\title{
Mice harbouring a SCA28 patient mutation in AFG3L2 develop late-onset ataxia associated with enhanced mitochondrial proteotoxicity
}

Cecilia Mancini $^{\mathrm{a}, 1}$, Eriola Hoxha ${ }^{\mathrm{b}, \mathrm{c}, 1}$, Luisa Iommarini $^{\mathrm{d}}$, Alessandro Brussino ${ }^{\mathrm{a}}$, Uwe Richter ${ }^{\mathrm{e}}$, Francesca Montarolo ${ }^{\mathrm{b}, \mathrm{c}}$, Claudia Cagnoli ${ }^{\mathrm{a}}$, Roberta Parolisi ${ }^{\mathrm{b}, \mathrm{c}}$, Diana Iulia Gondor Morosini ${ }^{\mathrm{b}, \mathrm{c}}$, Valentina Nicolò $^{\mathrm{b}, \mathrm{c}}$, Francesca Maltecca ${ }^{\mathrm{f}}$, Luisa Muratori ${ }^{\mathrm{c}, \mathrm{g}}$, Giulia Ronchi ${ }^{\mathrm{c}, g}$, Stefano Geuna ${ }^{\mathrm{c}, \mathrm{g}}$, Francesca Arnaboldi $^{\mathrm{h}}$, Elena Donetti ${ }^{\mathrm{h}}$, Elisa Giorgio ${ }^{\mathrm{a}}$, Simona Cavalieri ${ }^{\mathrm{a}}$, Eleonora Di Gregorio ${ }^{\mathrm{i}}$, Elisa Pozzi ${ }^{\mathrm{a}}$, Marta Ferrero ${ }^{\mathrm{a}}$, Evelise Riberi ${ }^{\mathrm{j}}$, Giorgio Casari ${ }^{\mathrm{f}}$, Fiorella Altruda ${ }^{\mathrm{k}}$, Emilia Turco $^{\mathrm{k}}$, Giuseppe Gasparre ${ }^{1}$, Brendan J. Battersby ${ }^{\mathrm{e}}$, Anna Maria Porcelli ${ }^{\mathrm{d}}$, Enza Ferrero ${ }^{\mathrm{a}}$, Alfredo Brusco $^{\mathrm{a}, \mathrm{i}, *, 1}$, Filippo Tempia ${ }^{\mathrm{b}, \mathrm{c}, 1}$

\footnotetext{
a Department of Medical Sciences, University of Torino, Torino, Italy

${ }^{\mathrm{b}}$ Department of Neuroscience, University of Torino, Torino, Italy

${ }^{\mathrm{c}}$ Neuroscience Institute Cavalieri Ottolenghi (NICO), Orbassano, Italy

${ }^{\mathrm{d}}$ Department of Pharmacy and Biotechnologies (FABIT), University of Bologna, Bologna, Italy

${ }^{\mathrm{e}}$ Institute of Biotechnology, University of Helsinki, Helsinki, Finland

${ }^{\mathrm{f}}$ Università Vita-Salute San Raffaele, Division of Genetics and Cell Biology, San Raffaele Scientific Institute, Milan, Italy

${ }^{\mathrm{g}}$ Department of Clinical and Biological Sciences, University of Torino, Torino, Italy

${ }^{\mathrm{h}}$ Department of Biomedical Sciences for Health, Università degli Studi di Milano, Milan, Italy

${ }^{i}$ Medical Genetics Unit, Città della Salute e della Scienza University Hospital, Torino, Italy

${ }^{\mathrm{j}}$ Department of Public Health and Pediatrics, University of Torino, Torino, Italy

${ }^{\mathrm{k}}$ Molecular Biotechnology Center, Department of Molecular Biotechnology and Health Sciences, University of Torino, Torino, Italy

${ }^{1}$ Department Medical and Surgical Sciences, Medical Genetics, University of Bologna, Bologna, Italy
}

\section{A R T I C L E I N F O}

\section{Keywords:}

SCA28

AFG3L2

Mouse knock-in

Mitochondrial dynamics

Proteotoxicity

\begin{abstract}
A B S T R A C T
Spinocerebellar ataxia 28 is an autosomal dominant neurodegenerative disorder caused by missense mutations affecting the proteolytic domain of AFG3L2, a major component of the mitochondrial m-AAA protease. However, little is known of the underlying pathogenetic mechanisms or how to treat patients with SCA28. Currently available $A f g 3 l 2$ mutant mice harbour deletions that lead to severe, early-onset neurological phenotypes that do not faithfully reproduce the late-onset and slowly progressing SCA28 phenotype. Here we describe production and detailed analysis of a new knock-in murine model harbouring an Afg3l2 allele carrying the p.Met665Arg patient-derived mutation. Heterozygous mutant mice developed normally but adult mice showed signs of cerebellar ataxia detectable by beam test. Although cerebellar pathology was negative, electrophysiological analysis showed a trend towards increased spontaneous firing in Purkinje cells from heterozygous mutants with respect to wild-type controls. As homozygous mutants died perinatally with evidence of cardiac atrophy, for each genotype we generated mouse embryonic fibroblasts (MEFs) to investigate mitochondrial function. MEFs from mutant mice showed altered mitochondrial bioenergetics, with decreased basal oxygen consumption rate, ATP synthesis and mitochondrial membrane potential. Mitochondrial network formation and morphology was altered, with greatly reduced expression of fusogenic Opa1 isoforms. Mitochondrial alterations were also detected in cerebella of 18-month-old heterozygous mutants and may be a hallmark of disease. Pharmacological inhibition of de novo mitochondrial protein translation with chloramphenicol caused reversal of mitochondrial morphology in homozygous mutant MEFs, supporting the relevance of mitochondrial proteotoxicity for SCA28 pathogenesis and therapy development.
\end{abstract}

\footnotetext{
* Corresponding author at: Department of Medical Sciences, University of Torino, via Santena 19, Torino 10126, Italy

E-mail address: alfredo.brusco@unito.it (A. Brusco).

${ }^{1}$ These authors equally contributed to the work.
} 


\section{Introduction}

The hereditary spinocerebellar ataxias (SCAs) are a group of over 40 neurodegenerative disorders characterized by autosomal dominant inheritance (Ashizawa et al., 2018). Although each form of SCA has its own distinct causative gene, the pathogenetic pathways converge on cerebellar and spinal degeneration leading to an array of slowly progressive neurological deficits (Ashizawa et al., 2018; Nibbeling et al., 2017; Smeets and Verbeek, 2014). We first described SCA type 28 (SCA28) over a decade ago in a four-generation Italian family with ataxia (Cagnoli et al., 2006; Di Bella et al., 2010; Mariotti et al., 2008), and later identified mutations in AFG3L2 (AFG3 ATPase Family Member 3-Like 2) as the cause of disease (Di Bella et al., 2010). Clinically, the age of onset ranges widely (from 3 to 76 years) and mean age of onset is 19.5 years. The principal manifestations are altered balance and gait, but limb ataxia, dysarthria and eye movement abnormalities have also been observed. The disease course is very slow, and patients remain ambulant into their late sixties. The principal finding by brain MRI is cerebellar atrophy, that usually appears as first hallmark of the disease (Brussino et al., 2018).

SCA28 is a rare disease, representing $\sim 1.5 \%$ of all autosomal dominant cerebellar ataxias (whose prevalence is 1-9/100,000). SCA28 has no treatment options, also because we lack a mechanistic understanding of disease pathogenesis. SCA28 is unique in being currently the only form of dominant ataxia caused by dysfunction of a mitochondrial-dwelling protein (http://neuromuscular.wustl.edu/ataxia/ domatax.html). The causative gene AFG3L2 is part of the nuclear genome and encodes a protein subunit that forms the $m$-AAA protease (matrix-ATPase associated with diverse cellular activities), a multimeric complex bound to the inner mitochondrial membrane (Koppen et al., 2007). The $m$-AAA protease is part of a complex network of evolutionarily conserved proteases that represent the most important inner defence system of mitochondrial integrity (Koppen and Langer, 2007; Patron et al., 2018), which is maintained also by other mechanisms such as regeneration or culling of compromised mitochondria by mitochondrial dynamics (fusion and fission) and removal of damaged organelles by mitophagy (Liesa et al., 2009). The m-AAA protease is a crucial component of the mitochondrial protein quality control system, exerting a chaperone-like activity during biogenesis of oxidative phosphorylation (OXPHOS) respiratory chain complexes. The $m$-AAA also participates in mitochondrial protein processing and maturation (Arlt et al., 1998; Atorino et al., 2003; Gerdes et al., 2012; Koppen and Langer, 2007; Nolden et al., 2005).

In humans, AFG3L2 is a major constituent of the m-AAA protease, being capable of self-aggregation to form the homo-hexameric form of m-AAA. AFG3L2 can also form hetero-hexamers in partnership with its paralogue SPG7 (alias paraplegin) (Koppen et al., 2007). To become active, both AFG3L2 and SPG7 themselves need to be proteolytically processed. However, while AFG3L2 is capable of self-activation, SPG7 requires AFG3L2 for maturation and activation via tyrosine phosphorylation, and does not self-assemble (Almontashiri et al., 2014; Koppen et al., 2009). Mutations in SPG7 are associated with the genetic disorder hereditary spastic paraplegia type 7 (Casari et al., 1998).

Structurally, the m-AAA protease is essentially an ATP-fueled proteolytic machine shaped like a double-doughnut: the juxtamembrane doughnut represents the ATPase domain while the proteolytic domain protrudes into the mitochondrial matrix (Koppen and Langer, 2007). Functionally, using as model the bacterial homologue $\mathrm{FtsH}$, we can imagine substrates being pulled through the hexamer ring of the AAA domain into the proteolytic cleft of the protease ring, with release of degradation peptides (Dalbey et al., 2012). Recently, Ding et al. demonstrated that a precursor form of the human MrpL32 (a mitoribosomal protein required for ribosomal assembly and protein synthesis) is targeted to AFG3L2, suggesting that m-AAA substrates may be evolutionary conserved (Almajan et al., 2012; Ding et al., 2018; Nolden et al., 2005).
We dwell on the complexities of the m-AAA structure, function and composition because it emerges that the majority of SCA28 patient mutations induce missense changes in AFG3L2 residues that participate in the formation of the proteolytic cleft. These missense mutations are mostly clustered in AFG3L2 exons 15 and 16 that form the protease domain: $\quad{ }^{654} \mathrm{Thr} \rightarrow \mathrm{Ile} ; \quad{ }^{666} \mathrm{Met} \rightarrow \mathrm{Val} / \mathrm{Arg} / \mathrm{Thr} ; \quad{ }^{671} \mathrm{Gly} \rightarrow \mathrm{Arg} / \mathrm{Glu}$; ${ }^{674} \mathrm{Ser} \rightarrow$ Leu; ${ }^{689} \mathrm{Tyr} \rightarrow$ Asn/His; ${ }^{691} \mathrm{Glu} \rightarrow \mathrm{Lys} ;{ }^{694} \mathrm{Ala} \rightarrow \mathrm{Glu} ;{ }^{700} \mathrm{Glu} \rightarrow$ Lys and ${ }^{702} \mathrm{Arg} \rightarrow$ Gln (Almajan et al., 2012; Cagnoli et al., 2010; Di Bella et al., 2010; Edener et al., 2010; Lobbe et al., 2014; Szpisjak et al., 2017; Zuhlke et al., 2015). Confirmation of the importance of modifications in the proteolytic cleft for disease pathogenesis comes from the ${ }^{432}$ Asn $\rightarrow$ Thr patient mutation: although encoded by exon 10 , thus outside of the mutational hotspot, it modifies a cleft-facing residue from the ATPase aspect. Two pathogenic frameshift mutations have also been described in SCA28: deletion of exons 14-16 and the p.Thr654Asnfs*15, again affecting the protease domain (Musova et al., 2014; Smets et al., 2014).

In addition to these observations on the nature and location of the patients' mutations, we note that the Exome Aggregation Consortium (ExAC) database (Lek et al., 2016) describes many human AFG3L2 variants (synonymous, missense, frameshift, gain of stop codon and altered splice sites) that are distributed throughout all the coding region. However, the loss-of-function AFG3L2 variants, whose frequency in the human population analysed is $\sim 1: 2000$ (i.e., far higher than the incidence of SCA28) are not deleterious, and ExAC gives a probability of LoF intolerance (pLI) of 0.01, indicating that AFG3L2 is extremely tolerant to loss-of-function changes, although it must be remembered that due to the late-onset of the phenotype, it is possible that some of the mutations have not had time to become manifest. It is evident that what is not tolerated is a select group of heterozygous missense mutations that do not appear to affect AFG3L2 protein levels but instead lead to incorporation of malfunctioning AFG3L2 subunits into the m-AAA complexes, which in turn become dysfunctional. AFG3L2 is highly expressed in cerebellum and other neural tissues, and our working hypothesis for SCA28 pathogenesis is that mitochondrial dysfunction in these tissues leads to proteotoxic stress through the slow accumulation of toxic misfolded substrates, a process already described in other neurodegenerative diseases such as Alzheimer's and Parkinson's diseases (Sorrentino et al., 2017). The ensuing mitochondriopathy eventually translates into neurodegeneration and neurological manifestations.

A mouse model of SCA28 would be an ideal approach to test our mitotoxicity hypothesis. Two murine mutations affecting Afg3l2 have already been described: one is the paralysé mouse ( $\left.A f g 3 l 2^{\text {par }} / \mathrm{par}\right)$, carrying the spontaneous Arg389Gly mutation in the conserved AAA domain, and the second is the Afg3l2 ${ }^{\text {Emv66/Emv66 }}$ mouse, in which Afg312 protein is absent because of a murine leukemia proviral insertion in intron 14 (Maltecca et al., 2008). Both mutants are smaller than normal littermates at one week, show a progressive loss of limb motor function by two weeks, and are completely paralyzed and usually die by 3 weeks. Mice present severe defects in neuronal development, with axonal development failure, aberrant mitochondria, Schwann cell invagination, and impaired maturation of Purkinje cell arborization (Maltecca et al., 2008). Compound heterozygotes (Afg3l2 ${ }^{\text {par/Emv66 }}$ ) are phenotypically indistinguishable from their respective homozygotes, suggesting that both mutations act by loss of a protective function (Maltecca et al., 2008). The models have proven valuable in deciphering the functional role of Afg312 in axonal development, where it is required for correct assembly of respiratory chain complexes (Maltecca et al., 2008). In contrast, heterozygous $A f g 3 l 2^{E m v 66 /+}$ mice have a milder phenotype, with the first signs of loss of balance appearing at four months and worsening with age, suggesting a dose-effect of the mutation (Maltecca et al., 2009). Additional informative models are the $S p g 7^{-{ }^{-}} A f$ $g 3 l 2^{E m v 6 /+}$ digenic mutated mice obtained by crossing the Afg3l2 $2^{E m v 66 /+}$ strain with SPG7 knock-out mice (Martinelli et al., 2009). These mice have a severe phenotype, characterized by early-onset loss of balance, 
tremor, and ataxia with altered gait coordination. Lastly, there is the Afg3l2 ${ }^{P C-K O}$ mouse, which limits Afg3l2 inactivation to Purkinje cells. Again, the phenotype is severe with unsteady gait at six weeks, caused by dramatic loss of Purkinje cells (Almajan et al., 2012). All these models serve to highlight the crucial role of Afg3l2 in maintaining normal cerebellar function, and that loss of Afg3l2 leads to disease with severe ataxic phenotypes. However, these models do not resolve the question of how the AFG3L2 missense mutations affecting the protease domain lead to SCA28.

With these considerations in mind, we hoped to generate a more authentic mouse model of disease by taking advantage of the fact that human and murine AFG3L2 proteins are highly conserved. This paved the way for knock-in generation by modifying one Afg3l2 allele through insertion of a SCA28 patient mutation. A potential drawback of this model is that rodent genomes harbour Afg3l1, a paralogue of $A f g 3 l 2$ and Spg7 that is pseudogenized in human (Kremmidiotis et al., 2001). However, Afg3l1 shows very low expression in brain and cerebellum (Sacco et al., 2010) and does not rescue the phenotype of either Afg3l2 or $S p g 7$ knock-out mice (Wang et al., 2016). Thus, we proceeded with our model, selecting the AFG3L2 missense mutation c.1994 T > G:p.Met666Arg (p.Met665Arg in mouse), reported in a familial case of SCA28 (Cagnoli et al., 2010). Here we describe: (i) the generation and motor behavior of the $A f g 3 l 2^{M 665 R}$ knock-in mouse; (ii) a detailed investigation of the effects of the mutation on neural tissues and their ultrastructure; (iii) a validation of the mitochondrial proteotoxicity model and (iv) that the antibiotic chloramphenicol can revert the mitotoxic phenotype, opening a potential new avenue of treatment.

\section{Materials and methods}

\subsection{Generation of Afg3l2 ${ }^{M 665 R /+}$ knock-in mice}

To generate our knock-in mouse model, we used a gene targeting approach to introduce the M665R mutation into Afg3l2. The targeting vector was constructed using the recombineering strategy (www. recombineering.ncifcrf.gov) (Copeland et al., 2001) and consisted of a $14.1 \mathrm{~kb}$ NotI-XhoI fragment from the BAC bmp360E12 (mouse strain 129) containing exon 16 and the flanking intron sequences (5261 bp upstream and $7128 \mathrm{bp}$ downstream) of murine $A f g 3 l 2$. In a second vector, we cloned a $0.6 \mathrm{~kb}$ region flanking exon 16 into which we introduced, through Sall-NotI restriction cloning, the neo ${ }^{r}$ cassette flanked by two Flippase recognition target sequences. The c.1994 T > G point mutation (p.Met665Arg) was introduced by site-directed mutagenesis (Stratagene) together with an $\mathrm{NcoI}$ restriction site at the $5^{\prime}$ end of exon 16 to allow discrimination between wild-type and mutated alleles by Southern blot. We used recombineering to generate the final vector for electroporation into mouse 129 ES cells. After positive selection with neomycin, 230 clones were harvested; 10 were positive by Southern blot using NcoI/SmiI-digested DNA. The Southern blot probe was a 300-bp fragment of Afg3l2 cloned into pUC19 after PCR amplification of mouse genomic DNA (primers $5^{\prime}$-ttcaggtctttcactggcatggt; $5^{\prime}$ gcaacacagtactatacactccttacg). The probed yields a $17.1 \mathrm{~kb}$ band in wild-type DNA, and $13.4 \mathrm{~kb}$ in mutant cells. The $n e o^{r}$ cassette copy number was evaluated by real-time PCR (Mancini et al., 2011). Only 2/ 10 ES cell lines showed a single neo $^{r}$ insertion and were injected into C57BL/ 6 blastocysts. The resulting chimeras were then mated to C57BL/6 females to obtain germline transmission. A diagnostic restriction enzyme digest was set up to verify the heterozygous presence of the mutation in mice: exon 16 was amplified using primers mAfg312_16F (5'-gctggtgcggtttgcccag) and mAfg312_16R (5'-cagcaggtagacactagctaagcaacc). Amplicons were digested with $H s p 92 I$ that cuts only the wild-type allele. The genotype of mice was confirmed by PCR, followed by Sanger sequencing. The neo ${ }^{r}$ cassette was eliminated by crossing Afg3l2 $2^{\text {M665R-neo/+ }}$ mice with a mouse strain containing the Flippase recombination enzyme. Afg $3 l 2^{M 665 R /+}$ animals were backcrossed with $\mathrm{C} 57 \mathrm{BL} / 6 \mathrm{~J}$ for at least seven generations to purify the genetic background. Studies were performed in accordance with the guidelines of the Neuroscience Institute Cavalieri Ottolenghi (NICO) (Orbassano, Italy), after obtaining approval by the Ethics Committee of the University of Torino, and following authorization from the Italian Ministry of Health (Authorization no.: 58/2016-PR).

\subsection{Assessment of motor phenotype}

Motor tests were performed every two months to detect the onset of neurological signs, starting when mice were 4 months old. The methods were as follows for 18 month old mice. The 18-month-old wild-type and Afg $312^{M 665 R /+}$ littermates were of similar weight (wild-type: $25.38 \pm 1.11 \mathrm{~g}, \quad n=11 ; \quad A f g 32^{M 665 R /+}: 25.98 \pm 0.62 \mathrm{~g}, \quad n=18 ;$ $\mathrm{P}>0.05)$. For the beam test (Goldowitz et al., 1992), Afg3l2 $2^{M 665 R /+}$ mice $(n=16)$ and their control littermates $(n=14)$ were placed on a metal beam ( $80 \mathrm{~cm}$ long $\times 1 \mathrm{~cm}$ wide) suspended above an open cage. Mice were allowed to traverse the beam for four days before the test (habituation). During the test, lateral slips were counted in three complete and consecutive crossings per day, on two consecutive days. The mean number of slips per $80 \mathrm{~cm}$ travelled was calculated. Footprint analysis was carried out using wild-type $(n=8)$ and $A f g 3 l 2^{M 665 R /}$ ${ }^{+}(\mathrm{n}=8)$ mice, as described in Hoxha et al. (Hoxha et al., 2013). Briefly, a cardboard tunnel was constructed on a transparent plexiglass platform ( $4 \mathrm{~cm}$ wide $\mathrm{x} 40 \mathrm{~cm}$ long). Video footage of mouse walks was obtained and analysed using ImageJ software. In the accelerating rotarod test, wild-type $(n=13)$ and $A f g 3 l 2^{M 665 R /+}(\mathrm{n}=13)$ mice were tested on five consecutive days, and on day 10. Each day, after $1 \mathrm{~min}$ training at constant speed ( $4 \mathrm{rpm})$, mice had three test sessions in which the rod (Mouse Rota-Rod, Ugo Basile Biological Research Apparatus, Comerio, Italy) accelerated continuously from 4 to $65 \mathrm{rpm}$ over $300 \mathrm{~s}$. The time to falling off the rod was recorded.

The optokinetic reflex (OKR) was measured to assess visual function. The OKR was evoked using a rotating optokinetic drum with an $8^{\circ}$ vertical grating. After placing the mouse on a central platform in the drum, and allowing $1 \mathrm{~min}$ for acclimation, the drum was rotated counterclockwise for $1 \mathrm{~min}$, stopped for $10 \mathrm{~s}$ and then rotated clockwise for another minute. Rotation velocity was $10^{\circ}$ s. The mouse was videorecorded and visual head tracking was measured offline (Thaung et al., 2002). Since the animal was free, there were episodes in which it spontaneously explored the environment, while in the remaining time the animal was still. Only the still-time was analysed (about $30 \mathrm{~s}$ ). The degree of optokinetic response was calculated as the percentage of time in which the mouse was still and tracked the images with the head relative to the total still-time.

\subsection{Histological analyses}

Eighteen-month-old $A f g 3 l 2^{M 665 R /+}(n=3)$ and wild-type $(\mathrm{n}=3)$ mice were anaesthetised by intraperitoneal injection with ketamine (100 mg/kg body weight) and xylazine $(10 \mathrm{mg} / \mathrm{kg}$ body weight), and perfusion-fixed with $4 \%$ paraformaldehyde in $0.12 \mathrm{M}$ phosphate buffer (PB). Brains were removed and immersed in the same fixative at $4{ }^{\circ} \mathrm{C}$ for $18 \mathrm{~h}$ and then cryoprotected in $30 \%$ sucrose in $0.12 \mathrm{M} \mathrm{PB}$. Cerebella were frozen and serially cut on a freezing microtome into $30 \mu \mathrm{m}$-thick sagittal sections. To label Purkinje cells, one set of sections was incubated overnight at $4{ }^{\circ} \mathrm{C}$ with anti-calbindin-D28k polyclonal antibody (CB-38a, 1:3000; Swant, Bellinzona, CH) diluted in PBS with $0.25 \%$ Triton X-100 and 1.5\% normal goat serum. Immunohistochemical reactions were stained with avidin-biotin-peroxidase (Vectastain $A B C$ Elite kit; Vector Laboratories, Burlingame, CA, USA) and revealed using $3,3^{\prime}$-diaminobenzidine $(0.03 \%$ in Tris- $\mathrm{HCl})$ as chromogen. A second set of sections was Nissl-stained to gauge the thickness of the molecular and granule cell cerebellar layers, and to quantify neuronal density (cell number $/ \mathrm{mm}^{2}$ ) in the deep cerebellar nuclei (DCN). Morphometric analysis of the cerebellar sections was performed by means of the Neurolucida system (MicroBrightField, Williston, VT, USA) and ImageJ 
software (http://rsbweb.nih.gov/ij/index.html).

\subsection{Purkinje cell firing}

Cerebellar slices were prepared as previously described (Llinas and Sugimori, 1980 and Edwards et al., 1989). Mice were anaesthetized with isoflurane (Isoflurane-Vet, Merial, Italy) and decapitated. The cerebellar vermis was removed and transferred to ice-cold artificial cerebrospinal fluid (ACSF) containing (in $\mathrm{mM}$ ); $125 \mathrm{NaCl}, 2.5 \mathrm{KCl}, 2$ $\mathrm{CaCl}_{2}, 1 \mathrm{MgCl}_{2}, 1.25 \mathrm{NaH}_{2} \mathrm{PO}_{4}, 26 \mathrm{NaHCO}_{3}, 20$ glucose, bubbled with $95 \% \mathrm{O}_{2} / 5 \% \mathrm{CO}_{2} \quad$ (pH 7.4). Parasagittal cerebellar slices $(200 \mu \mathrm{m}$ thickness) were obtained using a vibratome (Leica Microsystems $\mathrm{GmbH}$, Wetzlar, Germany) and kept for $1 \mathrm{~h}$ at $35^{\circ} \mathrm{C}$ and then at $31^{\circ} \mathrm{C}$. Single slices were placed in the recording chamber, which was perfused at a rate of $2-3 \mathrm{ml} / \mathrm{min}$ with ACSF bubbled with the $95 \% \mathrm{O}_{2} / 5 \% \mathrm{CO}_{2}$. All recordings were performed at $31 \pm 1{ }^{\circ} \mathrm{C}$ temperature. For cell-attached recording, the pipette was filled with $0.9 \% \mathrm{NaCl}, 0.2 \mu \mathrm{m}$ filtered. Recordings were made using an EPC-10 patch-clamp amplifier (HEKA Elektronik, Lambrecht/Pfalz, Germany). Gabazine (SR 95531, $20 \mu \mathrm{M}$ ), DAP5 $(50 \mu \mathrm{M})$ and NBQX $(10 \mu \mathrm{M})$ were added to the perfusate to inhibit the $\mathrm{GABA}_{\mathrm{A}}$ and ionotropic glutamate receptors of Purkinje cells, respectively. Data was derived from the analysis of 3-5 animals. All drugs were purchased from Abcam.

\subsection{Electron microscopy}

\subsubsection{Sciatic nerve}

To prepare sciatic nerve sections, 18-month-old wild-type mice $(n=5)$ and $A f g 32^{M 665 R /+}(\mathrm{n}=5)$ were anaesthetised by intraperitoneal injection with ketamine $(100 \mathrm{mg} / \mathrm{kg}$ body weight) and xylazine ( $10 \mathrm{mg} / \mathrm{kg}$ body weight). Using an operative microscope, the sciatic nerve was carefully exposed and removed. Nerves were immediately fixed by immersion in $2.5 \%$ glutaraldehyde in $0.1 \mathrm{M}$ phosphate buffer ( $\mathrm{pH} 7.4)$ for $4-6 \mathrm{~h}$ at $4{ }^{\circ} \mathrm{C}$. Samples were postfixed in $2 \%$ osmium tetroxide for $2 \mathrm{~h}$ and dehydrated in ethanol from $30 \%$ to $100 \%$. After two passages of $7 \mathrm{~min}$ each in propylene oxide and overnight in a 1: 1 mixture of propylene oxide and Glauerts' mixture of resins, sciatic nerves were embedded in equal parts of Araldite $M$ and the Araldite Harter resin, with further addition of $0.5 \%$ dibutylphthalate plasticizer. Finally, $2 \%$ of accelerator 964 was added to the resin in order to promote the polymerization of the embedding mixture.

\subsubsection{Light microscopy and design-based quantitative morphology of sciatic} nerves

From each nerve, 2.5- $\mu \mathrm{m}$-thick series of semi-thin transverse sections were cut using an Ultracut UCT ultramicrotome (Leica Microsystems, Wetzlar, Germany) and stained with 1\% Toluidine blue for high resolution light microscopic examination and design-based stereology. A DM4000B microscope equipped using a DFC320 digital camera and an IM50 image manager system (Leica Microsystems) was used for stereology of nerve fibers. On one randomly selected toluidine blue stained semi-thin section, the total cross-sectional area of the whole nerve was measured by light microscope and 12-16 sampling fields were selected by systematic random sampling (Geuna, 2000; Geuna et al., 2000; Larsen, 1998). In each sampling field, a two-dimensional dissector procedure, which is based on sampling the "tops" of fibers, was adopted in order to avoid the "edge effect" (Geuna, 2000). Using this procedure, the total number of myelinated fibers $(\mathrm{N})$ was estimated. In addition, both fiber and axon areas were measured, and the diameter of fiber (D) and axon (d) were calculated. These data were used to calculate myelin thickness [(D - d)/2], myelin thickness/axon diameter ratio $[(D-d) / 2 d]$, and axon/fiber diameter.

\subsubsection{Quantitative analysis of mitochondria in sciatic nerves}

Ultra-thin $(70 \mathrm{~nm})$ sections were cut from the sciatic nerves using the same ultramicrotome described for the high resolution light microscopy (above). Nerve sections were stained with saturated aqueous solution of uranyl acetate and lead citrate. Ultra-thin sections were analysed using a JEM-1010 transmission electron microscope (JEOL, Tokyo, Japan) equipped with a Mega-View-III digital camera and a SoftImaging-System (SIS, Münster, Germany) for computerized acquisition of the images. On one randomly selected ultra-thin section, 10-15 fields were selected using a systematic random sampling protocol, with a magnification of $\times 15,000$. In each sampling field, the number of impaired and unimpaired mitochondria was estimated as \%, based on their morphological features such as the shape of mitochondria, the morphology of the cristae and evidence of swelling.

\subsubsection{Cerebellar and lumbar spinal cord electron microscopy}

After excision of sciatic nerves, animals were perfused-fixed with $3 \%$ glutaraldehyde diluted in 0.1 M Sorensen phosphate buffer ( $\mathrm{pH} 7.4$ ) to prepare cerebellar and lumbar spinal cord sections. Bioptic fragments obtained by cerebellum and lumbar spinal cord were immersed in $3 \%$ glutaraldehyde diluted in $0.1 \mathrm{M}$ Sorensen phosphate buffer for $4 \mathrm{~h}$ at $4{ }^{\circ} \mathrm{C}$, washed $\mathrm{x} 3$ for $30 \mathrm{~min}$ in Sorensen phosphate buffer, and post-fixed in $1 \%$ Osmium Tetroxide. After dehydration through an ascending series of ethanol solutions, samples were embedded in Durcupan (Durcupan, Fluka, Milan, Italy). Ultra-thin sections were obtained with an Ultracut ultramicrotome (Reichert Ultracut R-Ultramicrotome, Leika, Wien, Austria) and stained with uranyl acetate/lead citrate before observation by a Jeol CX100 transmission electron microscope (Jeol, Tokyo, Japan).

\subsection{Caudal nerve conduction velocity}

To measure caudal nerve conduction velocity (NCV), mice were anaesthetised via intraperitoneal injection with ketamine $(100 \mathrm{mg} / \mathrm{kg}$ body weight) and xylazine $(10 \mathrm{mg} / \mathrm{kg}$ body weight). Stainless steel subdermal needle electrodes were used to deliver supramaximal stimulation with 0.05 millisecond impulses using an isolated stimulator (A-M System 2100, Sequim, WA, USA). Low frequency filters were set to $300 \mathrm{~Hz}$ and high frequency filters were set to $10 \mathrm{kHz}$. NCV in the tail nerve was assessed by placing needle electrodes in the tail, with the positive recording electrode at a few $\mathrm{cm}$ from the base of the tail. In proximal to distal direction, the distances from the first electrode were: negative recording electrode $0.5 \mathrm{~cm}$, ground 1.5 , negative stimulation electrode $4 \mathrm{~cm}$, positive stimulation electrode $4.5 \mathrm{~cm}$. The latencies of the potentials recorded after nerve stimulation were determined and the NCV was calculated accordingly. All neurophysiological determinations were performed under standard conditions and external body temperature was maintained at $34^{\circ} \mathrm{C}$ with a heating lamp.

\subsection{Mouse embryonic fibroblasts (MEF) production and analysis}

\subsubsection{Generation of MEFs}

Primary MEFs were established from embryos obtained on day E13.5 from wild-type, KI-hz Afg3l2 $2^{M 665 R /+}$ and KI-ho $A f g 3 l 2^{M 665 R / M 665 R}$ mice using a standard protocol (Sharpless, 2006) with immortalization by SV40 plasmid transfection followed by $300 \mu \mathrm{g} / \mathrm{ml} \mathrm{G} 418$ antibiotic selection (Schuermann, 1990).

\subsubsection{Analysis of mitochondrial morphology}

Mitochondrial network morphology was analysed using the mitoRED probe (Invitrogen) and live imaging confocal microscopy. An average of 150 cells was analysed for each experimental condition. Mitochondrial length was measured using ImageJ software tools. Experiments were repeated at least three times, and two operators independently analysed the images. The t-student test on raw data was to calculate significance $(\mathrm{p}<0.05)$.

\subsubsection{Western blot analysis}

Total cell or tissue lysates were prepared by protein extraction in 
RIPA buffer. Twenty $\mu \mathrm{g}$ of proteins were separated by electrophoresis on $4-12 \%$ Bis-Tris Protein Gels (Life Technologies). Anti-Afg3l2 (14631-1AP, polyclonal rabbit) and anti-Oma1 (17116-1AP, polyclonal rabbit, specific for pre-pro-Oma1 of $60 \mathrm{kDa}$ ) antibodies were purchased from Proteintech. Anti-Vinculin (Millipore, \#AB6039) or anti- $\beta$-actin (Lifespan, LS-C51570) were used as loading controls. For Opa1 band processing, we performed standard western blotting with total cell or tissue lysates prepared by protein extraction using a Triton-containing buffer (Maltecca et al., 2012). Twenty $\mu$ g (MEFs) and $5 \mu$ g (tissues) were separated by electrophoresis on $8 \%$ Bolt $^{\mathrm{TM}}$ Bis-Tris Plus Gels (Life Technologies). Anti-Opa1 antibody was purchased from BD Transduction Laboratories \#612606 (Franklin Lakes, NJ, USA). Anti-Viculin was used as loading control.

\subsection{Analysis of mitochondrial bioenergetics}

\subsubsection{Preparation of crude mitochondria}

Dissected tissues were promptly chopped and homogenized (30 strokes) in 10 volumes/g wet tissue of mannitol/sucrose buffer (220 mM D-mannitol, $70 \mathrm{mM}$ sucrose, $20 \mathrm{mM}$ HEPES, $1 \mathrm{mM}$ EDTA and $0.1 \%$ BSA, pH 7.2) in a glass-Teflon pestle. The crude homogenate was centrifuged at $500 \mathrm{~g}$ for $10 \mathrm{~min}$ at $4{ }^{\circ} \mathrm{C}$. The low-speed supernatant was centrifuged at $10,000 \mathrm{~g}$ for $10 \mathrm{~min}$ at $4{ }^{\circ} \mathrm{C}$. The resulting mitochondrial pellet was carefully re-suspended to about $10-20 \mathrm{mg} / \mathrm{ml}$ in mannitol/ sucrose buffer. Protein concentration was determined by Bradford's method (BioRad, Hercules, CA, USA) with BSA as the standard.

\subsubsection{Respiratory complex activity}

Mitochondrial respiratory chain complex activity was assayed and normalized for protein content and citrate synthase (CS) activity as previously described (Ghelli et al., 2013). All drugs used for biochemical analyses were from Sigma-Aldrich (St Louis, MO, USA).

\subsubsection{Native gel electrophoresis}

To analyse isolated native complexes, mitoplasts were suspended in a buffer containing $750 \mathrm{mM}$ aminocaproic acid, $50 \mathrm{mM}$ Bis-Tris, $\mathrm{pH}$ 7.0, and solubilized with $20 \%$ n-Dodecyl beta-D-maltoside (DDM), corresponding to a DDM/protein ratio of $2.5(\mathrm{~g} / \mathrm{g})$. The suspension was incubated on ice for $10 \mathrm{~min}$ and then centrifuged at $13,000 \mathrm{~g}$ for $15 \mathrm{~min}$ (Ghelli et al., 2013). Solubilized mitochondrial proteins $(80 \mu \mathrm{g})$ were separated on native $4-16 \%$ Bis-Tris Gel (Invitrogen), as detailed before (Ghelli et al., 2013). Gels were run as high resolution Clear Native- and Blue Native-PAGE (hrCN- and BN-PAGE). After electrophoresis, samples in CN-PAGE gels were processed for CI in-gel-activity (CI-IGA); samples in BN-PAGE gels were used for western blotting. Immunodetection was carried out by incubating blots with antibodies directed against the NDUFA9 subunit of CI (Thermo Fisher Scientific), SDHA subunit of CII (Thermo Fisher Scientific), UQCRC2 subunit of CIII (AbCAm, Cambridge, UK), and COXIV of CIV (AbCam, Cambridge, UK).

\subsection{Mitochondrial Respiration assay}

Oxygen consumption rate (OCR) in adherent cells was measured with by XFe24 Extracellular Flux Analyzer (Seahorse Bioscience, Billerica MA, USA), as previously described (Iommarini et al., 2014). Briefly, cells were seeded in XFe24 cell culture microplates at $2 \times 10^{4}$ cells/well in $200 \mu \mathrm{l}$ complete medium and incubated at $37^{\circ} \mathrm{C}$ in $5 \% \mathrm{CO}_{2}$ for $24 \mathrm{~h}$. Assays were initiated by replacing the growth medium in each well with $670 \mu \mathrm{l}$ of unbuffered DMEM-high glucose supplemented with $1 \mathrm{mM}$ Na-pyruvate pre-warmed at $37^{\circ} \mathrm{C}$. Cells were incubated at $37^{\circ} \mathrm{C}$ for $30 \mathrm{~min}$ to allow temperature and $\mathrm{pH}$ to equilibrate. After obtaining the OCR baseline measurement, $1 \mu \mathrm{g} / \mathrm{ml}$ oligomycin, $0.25 \mu \mathrm{M}$ FCCP $((2-$ [2-[4-(trifluoromethoxy)phenyl]hydrazinylidene]-propanedinitrile), $1 \mu \mathrm{M}$ rotenone and $1 \mu \mathrm{M}$ antimycin A were sequentially injected. At the end of each experiment, the medium was removed and protein content was determined by sulforhodamine B (SRB) assay. OCR data (pmol/ min) were normalized to SRB absorbance (Iommarini et al., 2014). First, the basal oxygen consumption rate (basal respiration) was measured. Oligomycin inhibited ATP synthase activity, which led to the development of a proton gradient that inhibited electron flux and revealed the state of the coupling efficiency. FCCP uncoupled the respiratory chain and revealed the maximal capacity for reducing oxygen. The spare respiratory capacity was calculated by subtracting the basal respiration from the maximal respiration. Finally, rotenone combined with antimycin A was injected to inhibit the flux of electrons through complexes I and III; the remaining oxygen consumption rate was primarily due to non-mitochondrial respiration.

\subsection{Mitochondrial ATP synthesis assay}

The rate of mitochondrial ATP synthesis was measured in digitoninpermeabilized cells as previously described (Ghelli et al., 2013). Briefly, after trypsinization, cells $\left(10 \times 10^{6} / \mathrm{ml}\right)$ were suspended in buffer ( $150 \mathrm{mM} \mathrm{KCl,} 25 \mathrm{mM}$ Tris-HCl, $2 \mathrm{mM}$ EDTA, $0.1 \%$ BSA, $10 \mathrm{mM}$ potassium phosphate, $0.1 \mathrm{mM} \mathrm{MgCl}_{2}, \mathrm{pH} 7.4$ ), maintained at room temperature for $15 \mathrm{~min}$, then incubated with $50 \mu \mathrm{g} / \mathrm{ml}$ digitonin until $90-100 \%$ of cells were positive by Trypan blue staining. Aliquots $\left(3 \times 10^{5}\right)$ of permeabilized cells were incubated in the same buffer as above in the presence of the adenylate kinase inhibitor $\mathrm{P}^{1}, \mathrm{P}^{5}$-di(adenosine- $\left.5^{\prime}\right)$ pentaphosphate $(0.1 \mathrm{mM})$ and $\mathrm{CI}$ substrates $(1 \mathrm{mM}$ malate plus $1 \mathrm{mM}$ pyruvate), CII substrate $(5 \mathrm{mM}$ succinate plus $1 \mu \mathrm{M}$ rote-

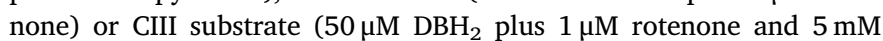
malonate). After the addition of $0.2 \mathrm{mM}$ ADP, chemiluminescence was determined as a function of time with a luminometer (Sirius-L, Berthold, Germany). The chemiluminescence signal was calibrated with an internal ATP standard after the addition of $1 \mu \mathrm{M}$ oligomycin. The rate of ATP synthesis was normalized to protein content and CS activity.

\subsection{Mitochondrial $\Delta \psi_{m}$ assay}

Mitochondrial $\Delta \psi_{\mathrm{m}}$ was assessed by FACS analysis, after incubating cells with $20 \mathrm{nM}$ tetra-methylrhodamine methyl ester perchlorate (TMRM) (Molecular Probes) for $30 \mathrm{~min}$ at $37^{\circ} \mathrm{C}$. After this, mitochondria were fully depolarized by addition of $5 \mu \mathrm{M}$ CCCP, as internal control. Data were analysed by WinList software (Verity Software House).

\subsection{Mitochondrial in vitro translation}

Mitochondrial protein synthesis was analysed in cultured cells by metabolic labelling with $\left[{ }^{35} \mathrm{~S}\right]$ methionine/cysteine in the presence of anisomycin to inhibit cytoplasmic ribosomes, as described in (Richter et al., 2015).

\section{Results}

\subsection{Generation of Afg3l2 ${ }^{M 665 R /+}$ knock-in mice}

A targeting vector for insertion of the c. $1994 \mathrm{~T}>\mathrm{G}$ (p.Met665Arg) missense mutation in Afg3l2 was prepared by recombineering (Copeland et al., 2001) (Fig. 1A). Briefly, the construct contained homologous DNA arms (C57BL/6 J mouse strain), a neo ${ }^{r}$ cassette and a diagnostic NcoI restriction site for Southern blotting. Following vector transfection into 129 embryonic stem (ES) cells, clones with one mutated allele and a single copy of neo ${ }^{r}$ were identified (Fig. 1B-C), and transferred to C57BL/6 blastocysts for germline transmission. $A f g 32^{M 665 R /+}$ knock-in (KI) mice were born at the expected Mendelian ratios, and were normal in terms of appearance, development and fertility. Expression of both KI and wild-type (WT) alleles was verified by RT-PCR and western blotting in different tissues, including cerebellum (Fig. 1D-F) and brain (data not shown).

When heterozygous mutant mice were intercrossed, the expected 
A

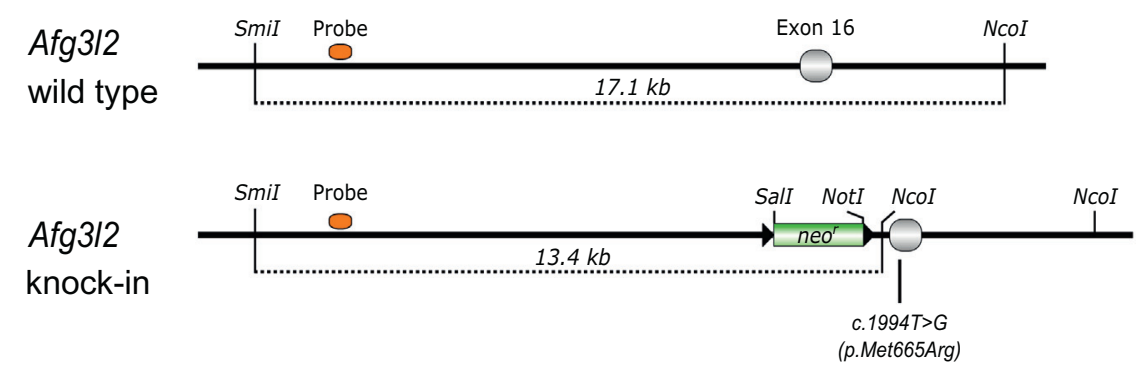

C

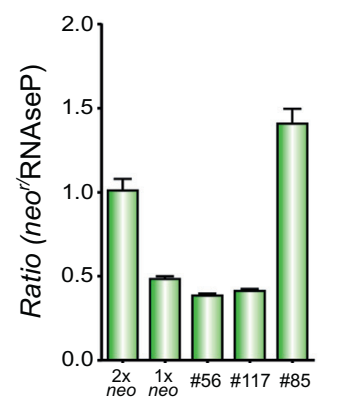

D

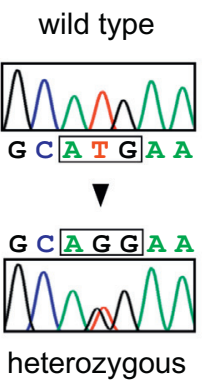

B

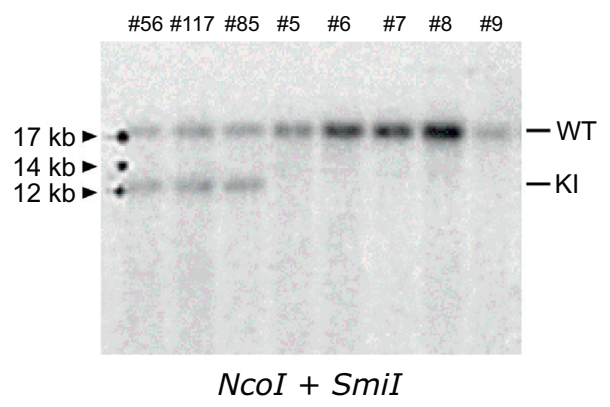

$\mathbf{F}$

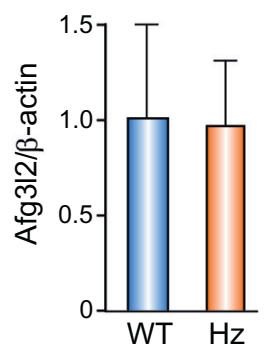

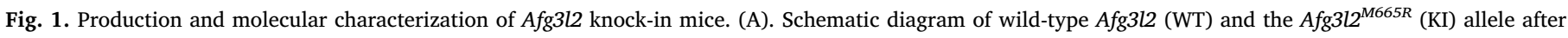

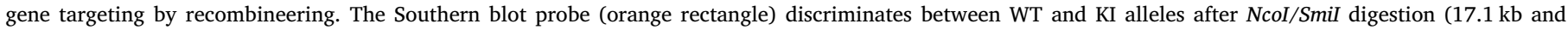

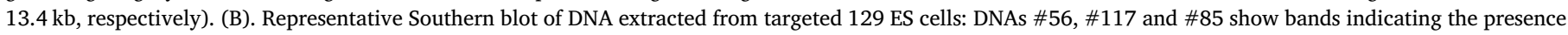

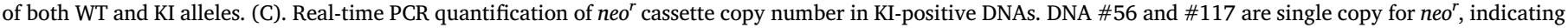

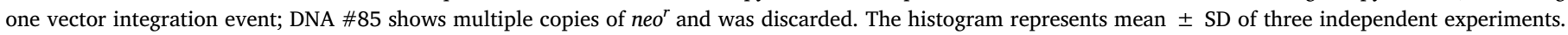

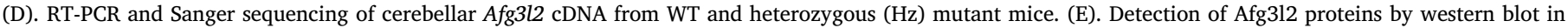

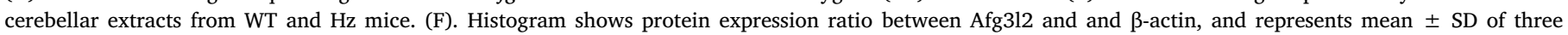
independent experiments.

Mendelian ratio at birth was skewed towards heterozygous and wildtype genotypes $\left(A f g 3 l 2^{+/+} 39 / 121, A f g 3 l 2^{M 665 R /+} 75 / 121\right.$, Afg3l2 ${ }^{M 665 R / M 665 R} 7 / 121$; Chi-square test, $\mathrm{p}=0.0003$ ). Most homozygous mutant mice died before or within hours of birth; rare viable mice were cyanotic. We obtained embryonic day 16.5 (E16.5) embryos for histological analyses (Supp. Fig. S1A); in $A f g 32^{M 665 R / M 665 R}$ embryos, the principal finding was diffuse cardiac atrophy while cerebellar histology appeared normal, although we cannot exclude a mild atrophy of the nervous system (Supp. Fig. S1B-E). The cardiac phenotype and perinatal lethality of the $A f g 322^{M 665 R / M 665 R}$ mouse contrasts with the Afg3l2 ${ }^{\text {Emv66/Emv66 }}$ mouse, which dies at around 2-3 weeks after birth with a neurological (tetraparetic) and cachectic phenotype (Maltecca et al., 2008), an indication that mutations that affect AFG3L2 quality $\left(A f g 32^{M 665 R / M 665 R}\right)$ and quantity $\left(A f g 3 l 2^{\text {Emv66/Emv66 }}\right)$ have different phenotypic effects. We return to the main focus, our hypothetical SCA28 model mouse, and leave further investigations into the cardiac phenotype of the homozygous mutant to future studies.

\subsection{Late-onset motor incoordination in Afg3l2 $2^{M 665 R /+}$ mice}

Although there was no outer evidence of disease, we started analysing motor behavior in Afg3l2 ${ }^{M 665 R /+}$ mice and wild-type littermates at 4 months so as not to miss the onset of motor alterations. We took as a guide the fact that, at this age, $A f g 3 l 2^{E m v 66 /+}$ mice show signs of motor incoordination. From the age of 4 months onwards, motor testing was repeated every two months. The test battery included: (i) the elevated beam test; (ii) footprint analysis and (iii) the accelerating rotarod.

When first examined ( 4 months), the tests showed no significant differences between wild-type and heterozygous mutants. However, at 18 months, Afg3l2 $2^{M 665 /+}$ mice started to show signs of imbalance on the beam test, with significantly greater number of fore paw and hind paw slips compared to wild-type littermates. Similar results were obtained when tests were repeated on consecutive days (Fig. 2A). In contrast, no differences were observed between groups of mice in footprint analysis (Fig. 2B), in the accelerating rotarod (Fig. 2C) nor in the day 10 rotarod test (Fig. $2 \mathrm{C}$ ), which measured motor memory retention. Since optic atrophy has been associated with AFG3L2 mutations (Charif et al., 2015; Colavito et al., 2017), the optokinetic response to motion of the visual surroundings was assessed in Afg $312^{M 665 R /+}$ and wild-type mice at 18 months. No significant difference in head tracking performance was observed between genotypes: tracking time with respect to total still-time was $81.4 \pm 4.41 \%$ in wildtype mice, and $90.9 \pm 2.10 \%$ in heterozygous mutants, ruling out vision loss. The test battery was repeated at 20, 22 and 24 months of age, with no evidence of increasing severity of the phenotype (data not shown). Thus, the heterozygous p.Met665Arg in Afg312 appears to be a mild mutation leading to adult-onset ataxia in mice that well recapitulates the SCA28 patient phenotype.

\subsection{Ataxic Afg3l2 $2^{M 665 R /+}$ mice show normal cerebellar structure}

Cerebellar degeneration is central to ataxic neuropathology and usually precedes the onset of motor dysfunction. Therefore, we began examining cerebellar structure in heterozygous mutant mice from 6 months onwards, with no pathological finding. Even in 18-month-old ataxic heterozygotes, Nissl-stained cerebellar sections showed regular lamination and foliation as in control mice (Fig. 2D, E). Molecular and granular layer thickness was quantified in all cerebellar lobules, using a previously described method (Carulli et al., 2002), with no evident differences between genotypes (Fig. $2 \mathrm{H}, \mathrm{I}$ ).

Since AFG3L2 is required for cerebellar Purkinje cell survival (Almajan et al., 2012), Purkinje cell numbers were estimated by 
A

Balance Beam Test

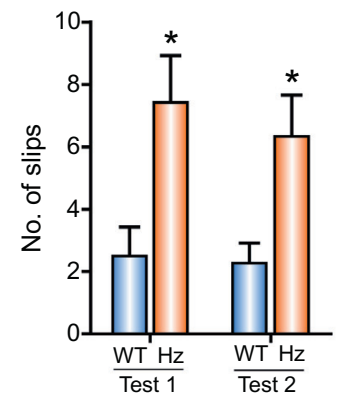

WT
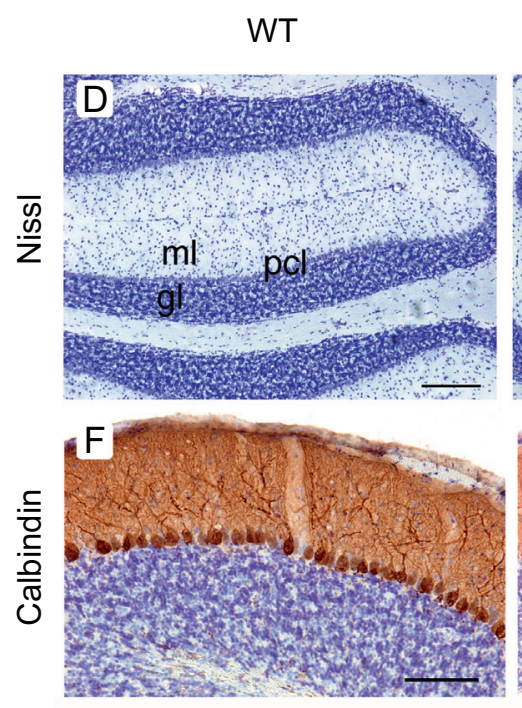

B

Footprint Test

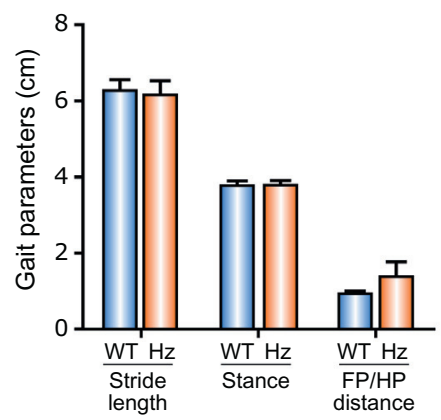

$\operatorname{Afg} 3 / 2^{M 665 R /+}$
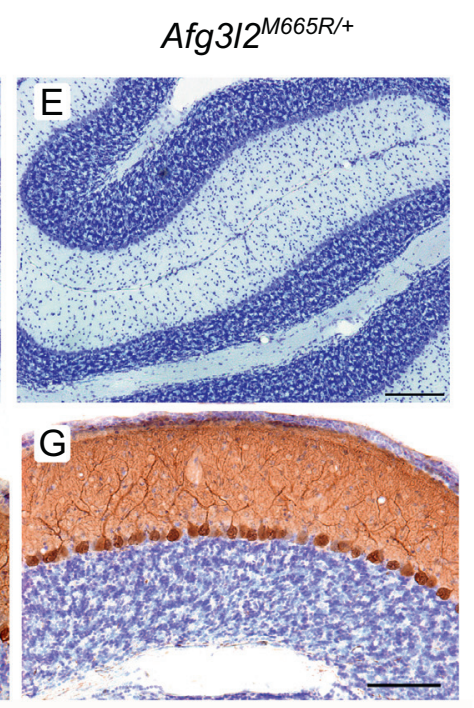

C

Accelerated Rotarod Test

\section{Cerebellum}

$\mathrm{K}$
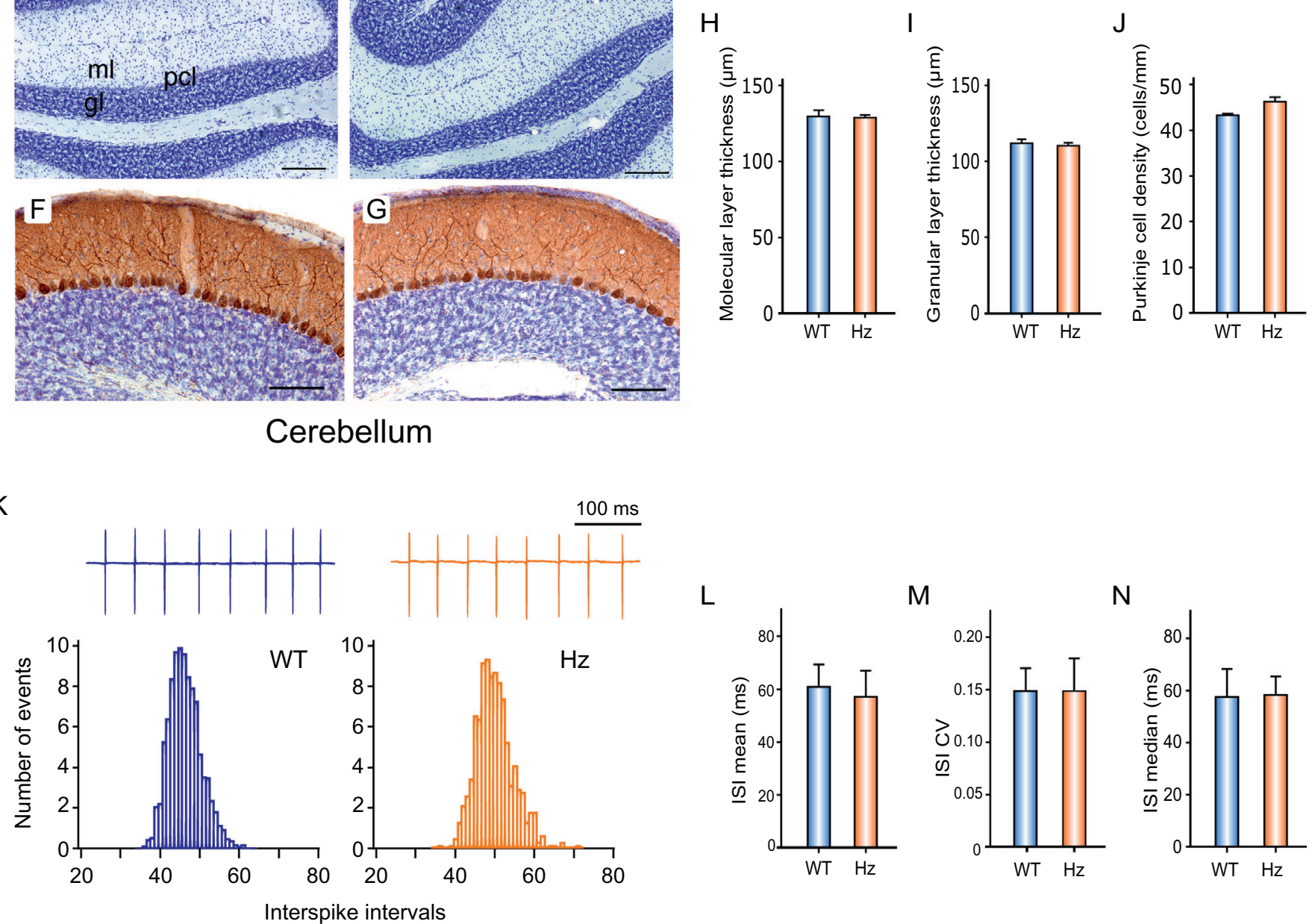

L

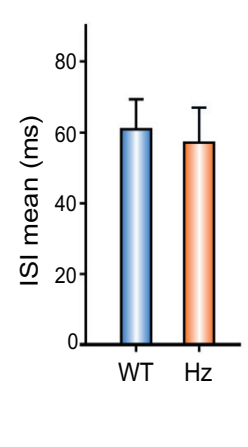

M

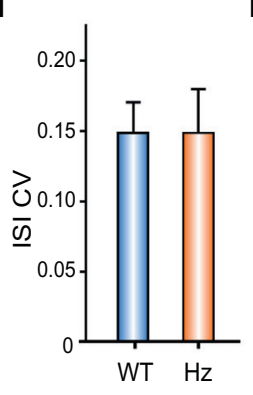

$\mathrm{N}$

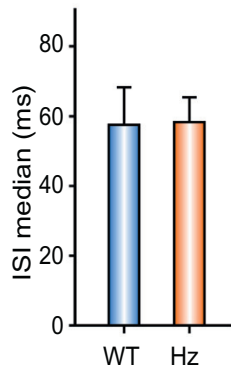

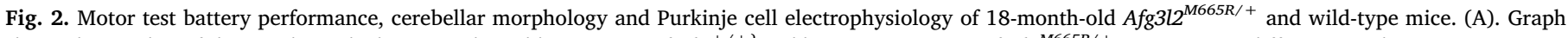

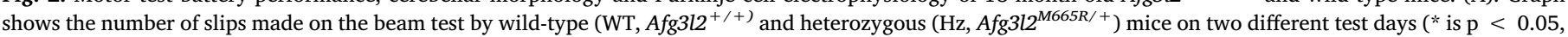

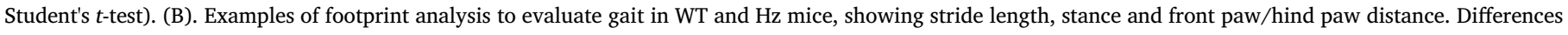

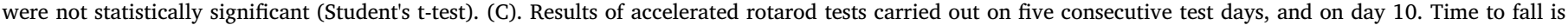

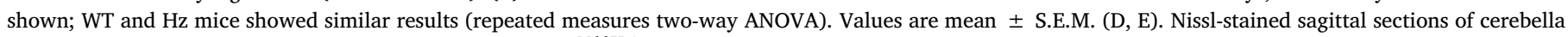

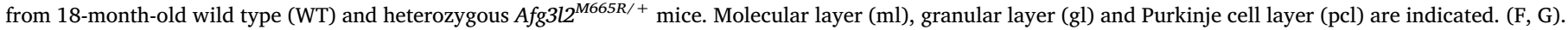

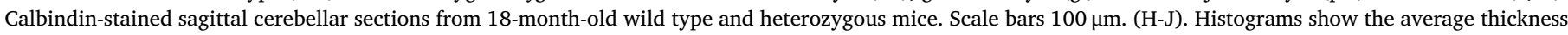

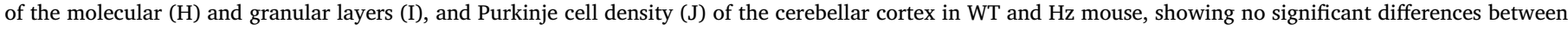

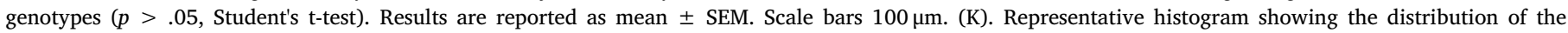

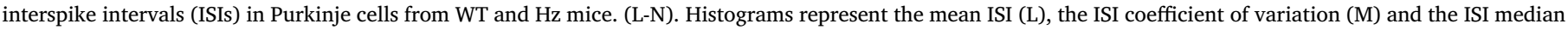

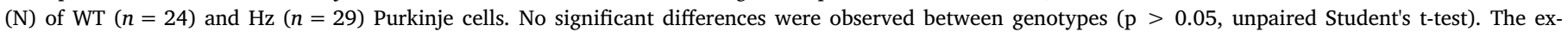
periment has been repeated on a second set of Purkinje cells, which confirmed the absence of a significant difference. 
calbindin staining in cerebellar sagittal sections (Fig. 2F-G). The average number of Purkinje cells was similar in $A f g 3 l 2^{\mathrm{M} 665 R /+}$ (46.2 \pm 0.94 cells $/ \mathrm{mm}, n=3$ ) and wild-type mice ( $43.2 \pm 0.20$ cells/ $\mathrm{mm}, \mathrm{n}=3$ ) (Fig. $2 \mathrm{~J}$ ). Both groups also showed similar cell numbers in the deep cerebellar nuclei (data not shown).

\subsection{Ataxic Afg3l2 $2^{M 65 R /+}$ mice show normal spontaneous firing activity in Purkinje cells}

We next investigated $A f g 3 l 2^{M 665 R /+}$ Purkinje cell function by measuring the generation of spontaneous action potentials. Individual Purkinje cells in cerebellar slices were analysed by cell-attached patch clamp recordings. The mean interspike interval (ISI) was not significantly different in Purkinje cells from 18-month-old wild-type and heterozygous mutant mice (Fig. 2K-L). The regularity of Purkinje cell firing was also conserved, as shown by the ISI coefficient of variation (Fig. 2M). The median of the interspike intervals was also similar for both genotypes (Fig. 2N). However, we did note an increase in the number of firing cells in $A f g 3 l 2^{M 665 R /+}$ mice, but this was not statistically significant - WT Purkinje cells: 47 analysed/21 silent/26 (55\%) firing; Hz Purkinje cells: 36 analysed/9 silent/27 (75\%) firing.

\subsection{Normal neural ultrastructure but alterations in sciatic nerve mitochondria in Afg3l2 $2^{M 665 R /+}$ mice}

Previously described Afg3l2 mutant mouse models show altered mitochondrial morphology in cells of the nervous system (Maltecca et al., 2008; Maltecca et al., 2009). Therefore, we carried out detailed ultrastructural analyses of CNS- and PNS-derived tissues.

\subsubsection{Cerebellum}

In 18-month-old wild-type and heterozygous mutant mice, the cerebellar cortex showed the typical molecular, Purkinje and granular layer organization, with clear boundaries between layers. Purkinje cells were aligned in monolayers, with cell bodies near the granular layer, a large nucleus within the piriformis-shaped soma (Fig. 3A-B), and dendritic arborisations that crossed the molecular layer. No cell body shrinkage, enlargement of the ER cisternae nor fragmentation of the Golgi apparatus was observed. Mitochondria were normal with evident cristae and no signs of swelling (Fig. 3C-D). The granular layer was packed with small round cells (Fig. 3E-F).

\subsubsection{Spinal cord}

Spinal cord white matter was similar in wild-type and $A f g 3 l 2^{M 665 R /}$ ${ }^{+}$mice: myelinated nerve fibers of small, medium and large diameter were ubiquitous, and myelinated sheaths showed uniform thickness (Fig. 3G-H). Axonal mitochondria always displayed conserved cristae, homogeneous matrix, and no signs of swelling (Fig. 3I-J).

\subsubsection{Sciatic nerve}

Sciatic nerves were isolated and examined by high-resolution light microscopy. Nerve fibers showed similar organization and morphology, and similar size in axon diameter, fiber diameter and myelin sheaths (Fig. 3K, L). Sciatic nerve fiber morphology by stereological analysis of myelinated fibers showed no significant differences between groups in the total number of myelinated fibers, axon diameter, fiber diameter and myelin thickness (Fig. 3M-P). Increased numbers of sciatic nerve mitochondria from Afg3l2 ${ }^{M 665 R /+}$ mice showed altered cristae morphology and signs of swelling compared to wild-type, by stereological analysis: altered mitochondria in wild-type: $6.39 \pm 1.97 \%$; altered mitochondria in $A f g 3 l 2^{M 665 R /+}: 43.1 \pm 4.98 \%$. (p $<0.0001$, Chisquared test) (Fig. 3Q, R). However, there were no discernible differences in peripheral nerve conduction of action potentials between groups: wild-type $(n=7) 32.7 \pm 1.2 \mathrm{~m} / \mathrm{s} ; A f g 32^{M 665 R /+}(n=6)$ $32.9 \pm 1.7 \mathrm{~m} / \mathrm{s}$.

\subsection{Mitochondrial bioenergetics are altered in Afg3l2 ${ }^{M 665 R /+}$ mouse embryonic fibroblasts}

To analyse the impact of the p.Met665Arg mutation on mitochondrial function, we generated mouse embryonic fibroblasts (MEFs) from E13.5 embryos as these were available for the wild-type and mutant $\left(A f g 3 l 2^{M 665 R /+}\right.$ and $A f g 3 l 2^{M 665 R / M 665 R}$ ) genotypes. MEFs were then used to analyse mitochondrial dynamics, bioenergetics, and oxidative phosphorylation (OXPHOS). Compared to wild-type MEFs, Afg3l2 $2^{M 665 /}$ + and $A f g 3 l 2^{M 665 R / M 665 R}$ MEFs showed significant reduction in: (i) basal oxygen consumption rate; (ii) maximum respiratory capacity; (iii) spare respiration reserve and (iv) ATP-linked respiration, evaluated using the Seahorse system (Fig. 4A; Supp. Fig. S2A). In addition, Afg3l2 $2^{M 665 R /+}$ MEFs showed reduced extracellular acidification rates, indicating reduced metabolic activity compared to controls (Fig. 4A; Supp. Fig. S2A). The mitochondrial membrane potential $(\Delta \Psi \mathrm{m})$ was significantly decreased in $A f g 3 l 2^{M 665 R / M 665 R}$ MEFs (Fig. 4B) compared to wild-type at steady state, pointing to a defect in the proton pump possibly caused by impaired function of OXPHOS complexes.

Reduced ATP-linked respiration was confirmed by ATP synthesis assay performed in digitonin-permeabilized cells. Homozygous mutant MEFs exhibited a significant decrease of Complex I-driven and Complex II-driven ATP synthesis, indicating that the p.Met665Arg Afg312 variant impairs respiratory complex chain activity (Fig. 4C). By spectrophotometric analysis of isolated mitochondrial fractions, we found normal levels of activity in Complex I, II and IV in both $A f g 312^{M 665 R /+}$ and $A f g 3 l 2^{M 665 R / M 665 R}$ MEFs. However, there was a $30 \%$ reduction of Complex III activity, which was statistically significant, in mitochondria isolated from $A f g 3 l 2^{M 665 R / M 665 R}$ MEFs. Citrate synthase activity was comparable in all three genotypes (Fig. 4D-E), indicating that there were no overall differences in mitochondrial mass.

To evaluate mitochondrial protein maturation together with the assembly and stability of OXPHOS complexes, we analysed mitochondrial in vitro translation, the steady-state levels of selected OXPHOS complex subunits and of fully assembled respiratory complexes by native PAGE. No differences were found between MEFs in terms of de novo mitochondrial protein synthesis assessed in organello after incorporation of ${ }^{35} \mathrm{~S}$-Met/Cys (Supp. Fig. S2B), indicating proper synthesis of mitochondrial proteins in the presence of mutant Afg312. The steady-state levels of respiratory complex subunits in MEFs were similar for all genotypes (Supp. Fig. S2C). Fully assembled Complex III was reduced in Afg3l2 $2^{M 665 R / M 665 R}$ MEFs, possibly explaining the reduced Complex III redox activity found in these cells, whereas the other respiratory complexes were expressed in normal amounts (Supp. Fig. 2D).

\subsection{Altered mitochondrial dynamics induced by the p.Met665Arg mutation in MEFs and mutant mice}

To determine the impact of the p.M665R mutation on mitochondrial morphology, MEFs were treated with MitoRed to visualize fluorescently stained mitochondria. Afg3l2 $2^{\text {M665R/M665R }}$ MEFs showed fragmented mitochondria, reminiscent of observations made in both $A f g 32^{\text {Emv66/ }}$ Emv66 MEFs and mutant mice (Maltecca et al., 2012) whereas Afg $312^{M 665 R /+}$ MEFs showed intermediate/tubular mitochondria (Fig. 5A, B).

MEF expression of proteins that regulate mitochondrial fission and fusion were analysed by western blot. The GTPase Opa1 (Optic atrophy 1 ) is a major regulator of mitochondrial morphology: long isoforms (LOpa1) promote mitofusion whereas the proteolytically-generated short forms (S-Opa1) instead trigger mitochondrial fragmentation and fission. Afg3l2 ${ }^{M 665 R / M 665 R}$ MEFs showed a remarkable decrease in L-Opa1, pointing to increased proteolytic processing to S-Opa1 (Fig. 5C). We also analysed expression of Drp1 (pro-fission) and the mitofusins Mfn1 and Mfn2 in MEFS from the three genotypes, but found no major differences in expression (Supp. Fig. S2E).

To return to our mouse model, we next evaluated Opa1 expression 

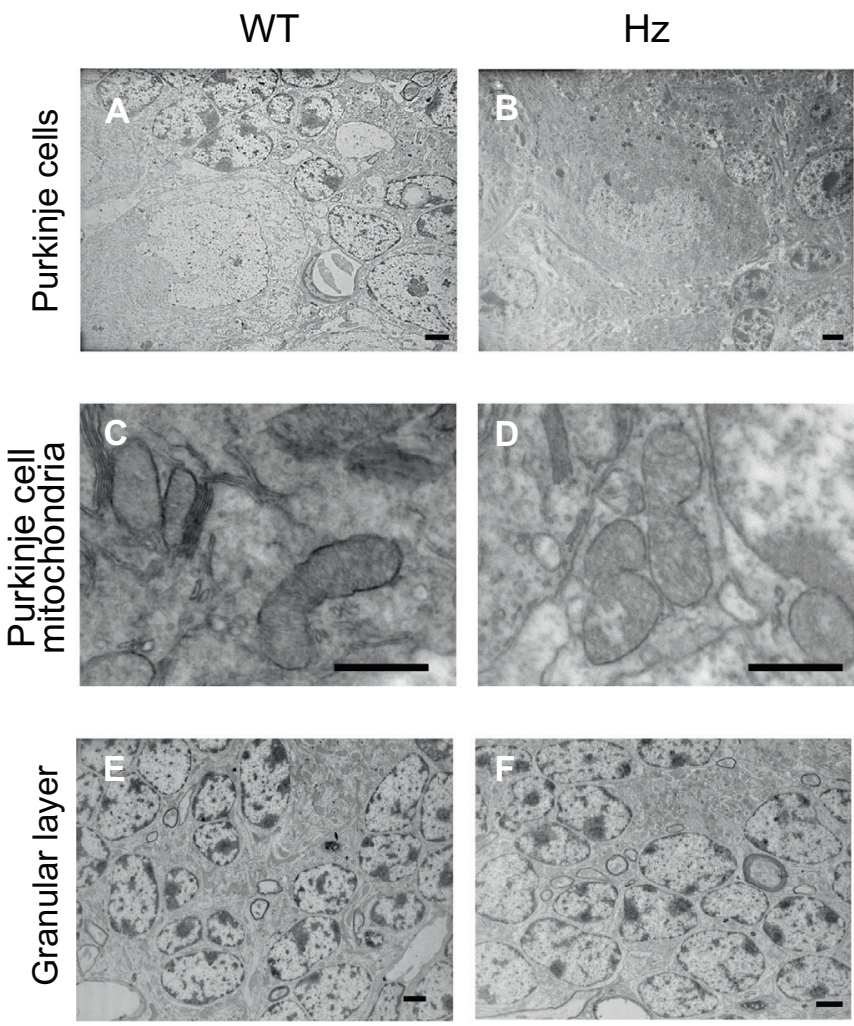

Cerebellum

M

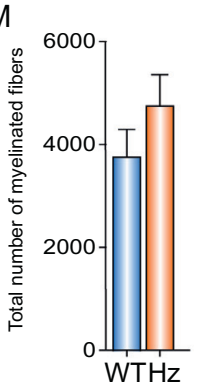

$N$

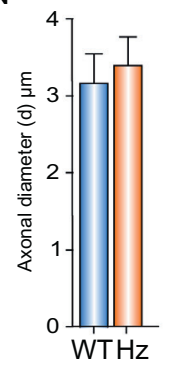

$\mathrm{Hz}$
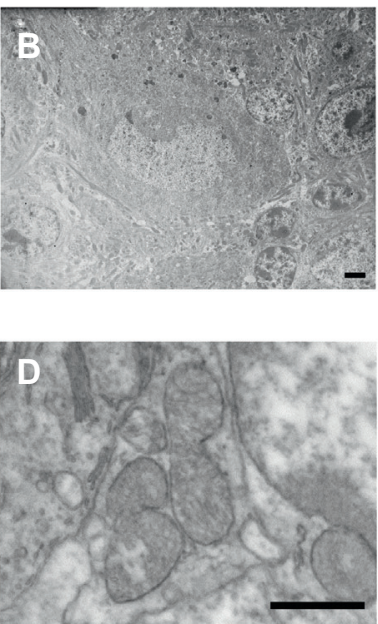

O

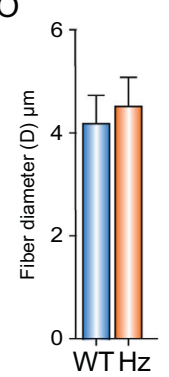

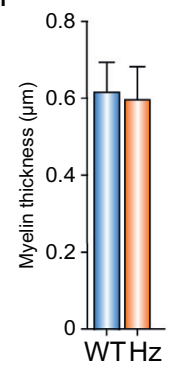

WT
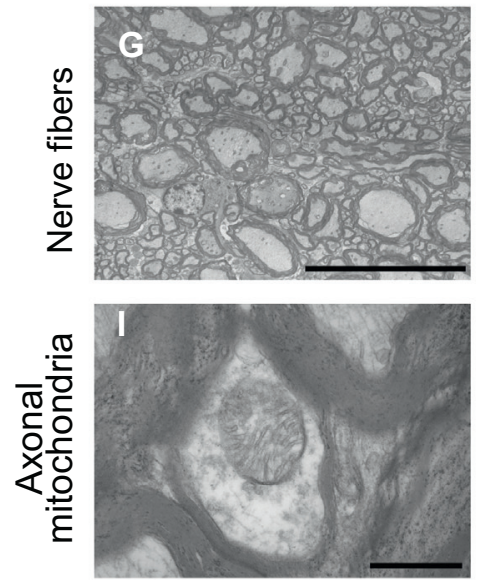

Spinal cord

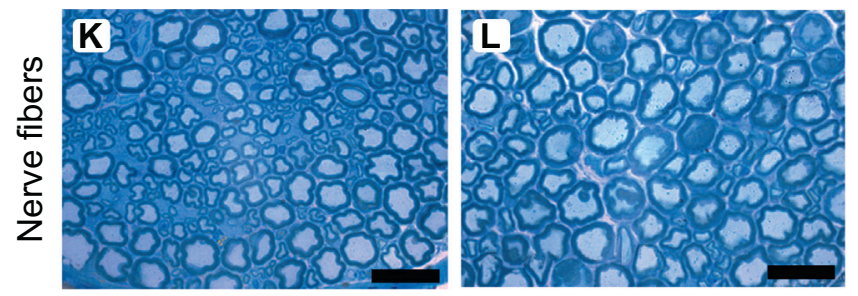

Sciatic nerve

$\mathrm{Hz}$
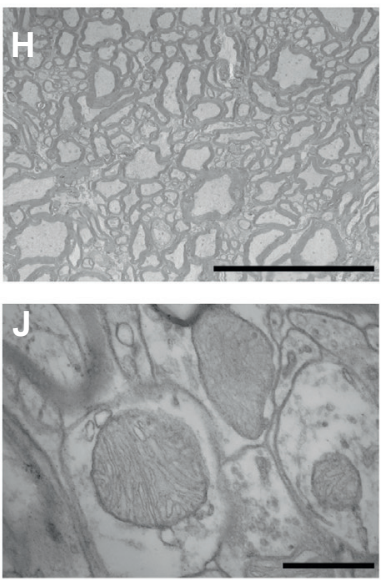

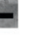




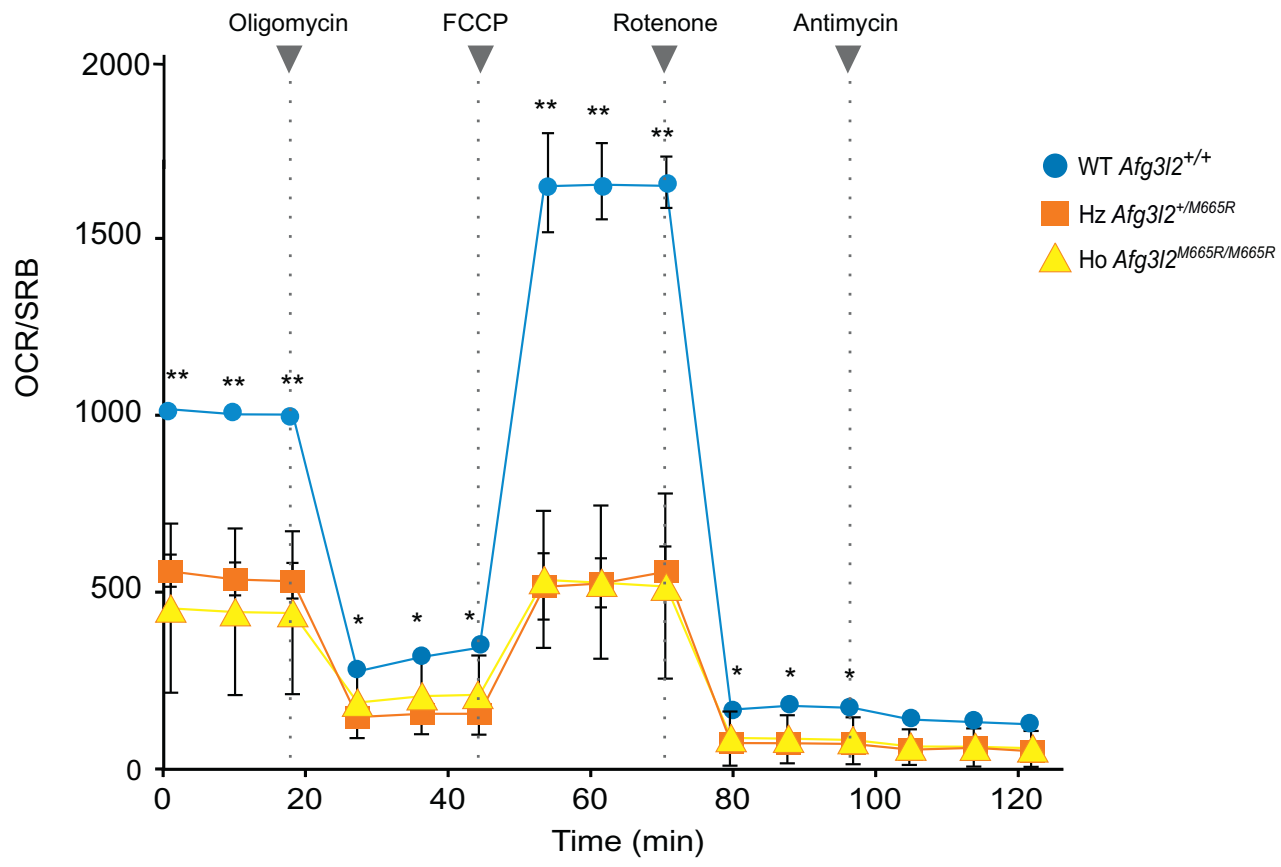

B

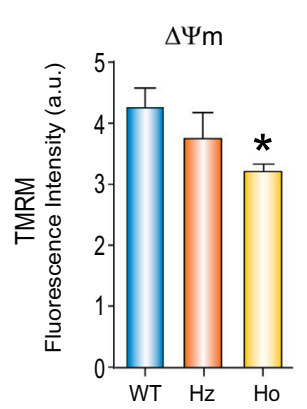

E

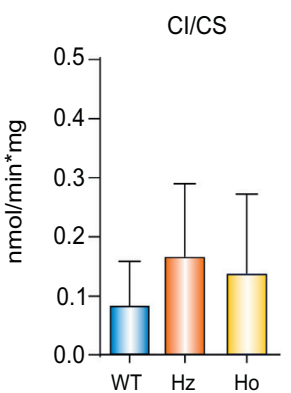

C
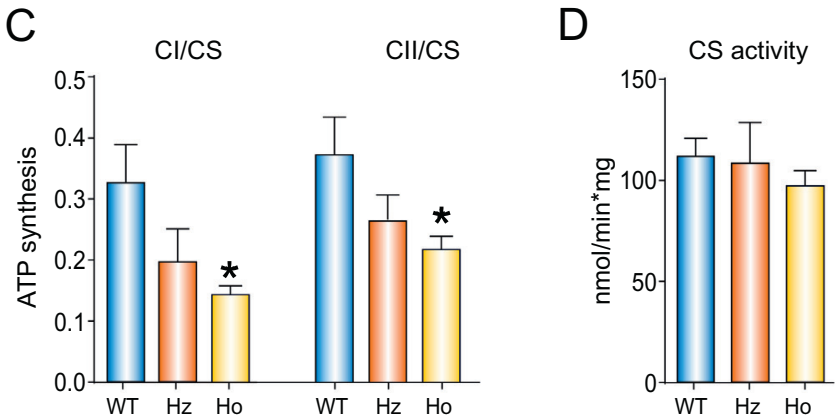
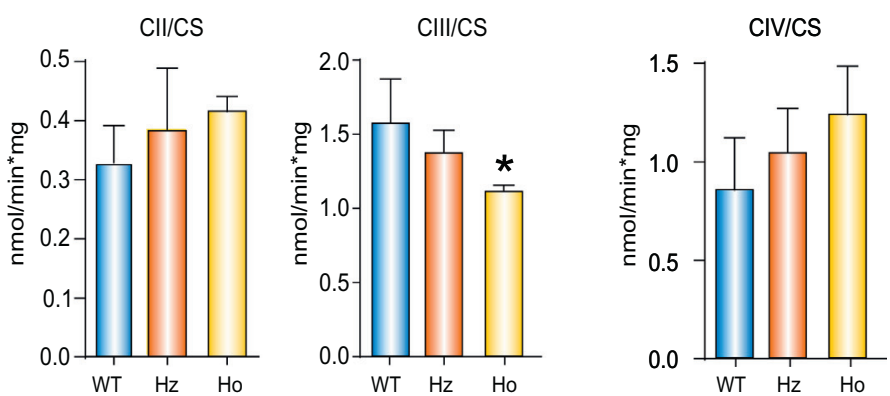

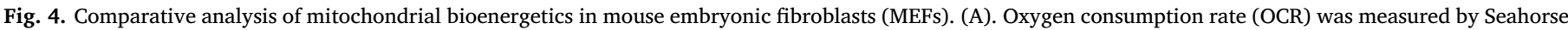

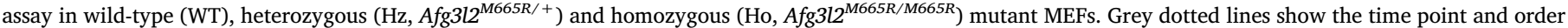

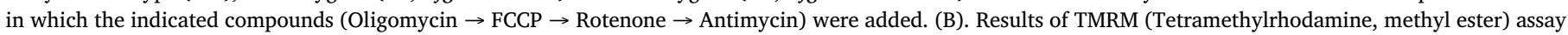

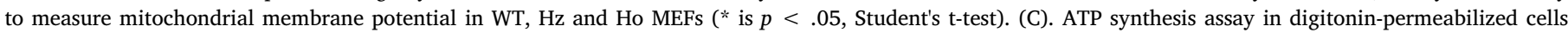

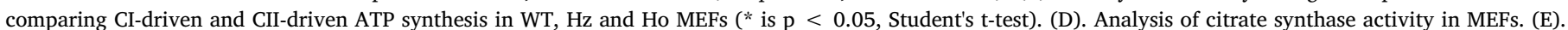

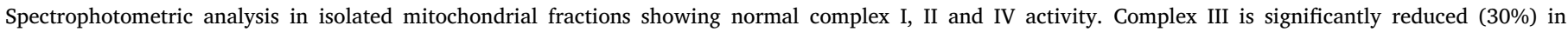
homozygous (Ho) mutants with respect to WT and $\mathrm{Hz}$ (* is $\mathrm{p}<0.05$, Student's t-test). 
A

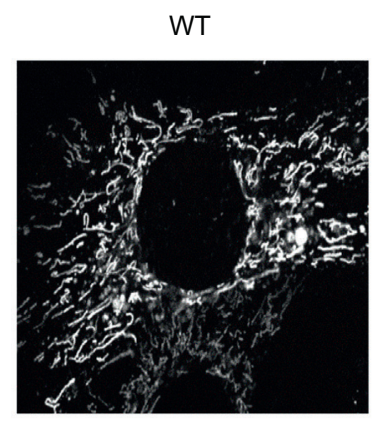

B

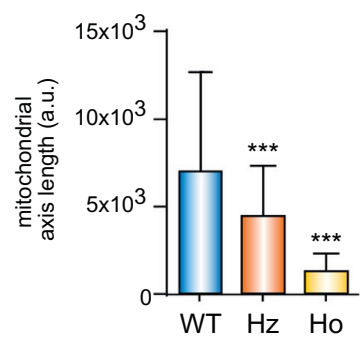

$\operatorname{Afg} 3 / 2^{M 665 R /+}$

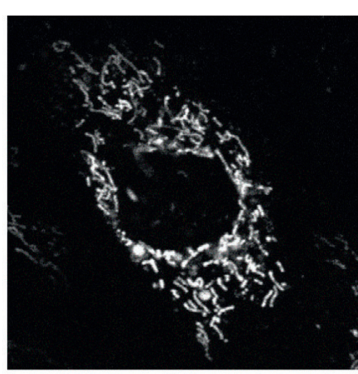

MEFs + MitoRed

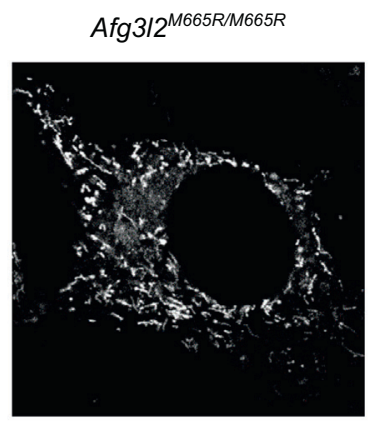

C

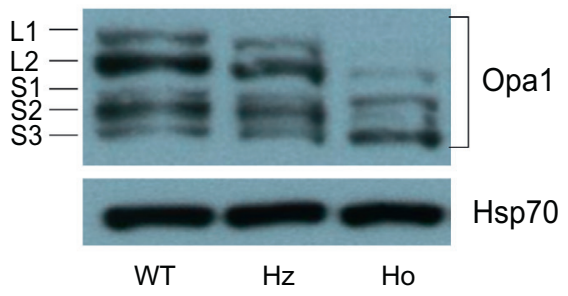

D
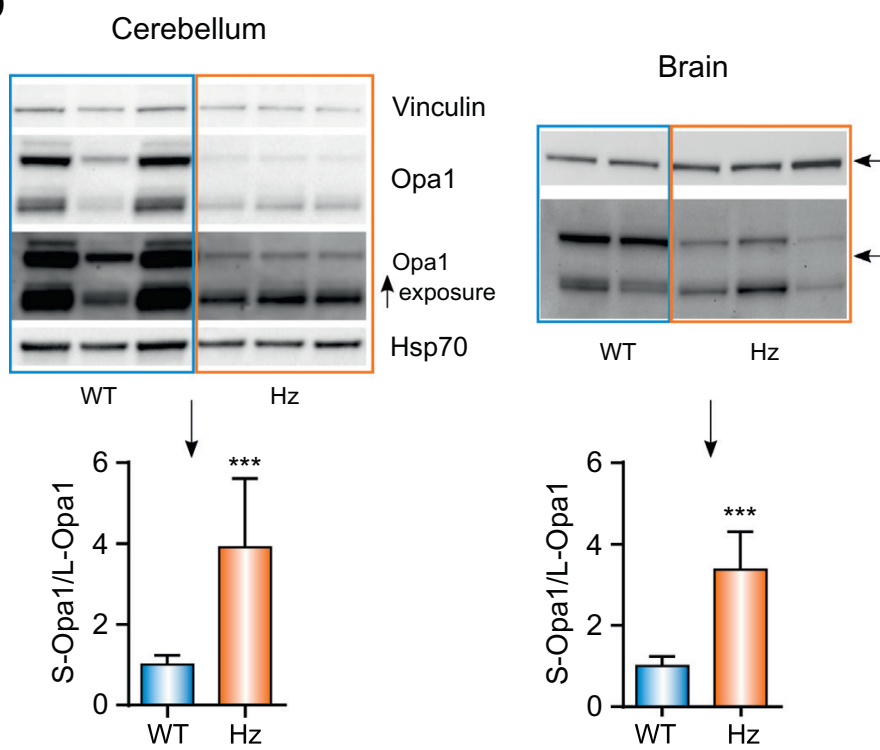

Optic nerve

Fig. 5. Mitochondrial dynamics in MEFs.

(A). Representative images obtained by live confocal microscopy showing mitochondrial morphology in wild-type, $A f g 32^{M 665 R /+}$ (Hz) and $A f g 322^{M 665 R / M 665 R}$ (Ho) MEFs after staining with mitoRED fluorescent probe. (B). Morphometric analysis of mitochondrial morphology: mitochondria from 50 MEF cells per genotype were measured along the length of the major mitochondrial axis. Histograms represent mean \pm SD of four independent experiments (a.u., arbitrary units). WT vs. either $\mathrm{Hz}$ or Ho $(* * *$ is $\mathrm{p}<0.0001$, Student's t-test, two-tailed). (C). Immunoblot of MEF total lysates stained for Opa1; Hsp70, loading control. (D). Immunoblot analysis of Opa1 processing in homogenates of neuronal tissues from WT and heterozygous Afg3l2 ${ }^{M 665 /+}$ mice. Histograms below gels show the ratio between short and long Opa1 isoforms.

generating the stress-sensitive form, pro-OMA1 (40 kDa). This in turn is cleaved to generate the active form of OMA1, which is responsible for OPA1 processing (Baker et al., 2014). Very recently, AFG3L2 has been shown to play a major role in converting pre-pro-OMA1 to pro-OMA1 (Consolato et al., 2018). A consequence of AFG3L2 malfunction is that mitochondrial protein synthesis leads to OMA-1 activation (Arlt et al., 1996; Richter et al., 2015). Similarly, we expected mitochondrial protein synthesis to perturb mitochondrial quality control in our mutant mouse model bearing dysfunctional m-AAA complexes containing Afg312 p.Met665Arg subunits. Prokaryotic and mitochondrial protein synthesis machineries are similar, so antibiotics such as chloramphenicol (CAM) that target bacterial ribosomes often affect mitochondrial protein synthesis too. We tried blocking mitochondrial protein synthesis in MEFs with CAM: remarkably, $24 \mathrm{~h}$ CAM treatment completely restored L-Opa1 levels to normal in homozygous mutant MEFs (remaining elevated up to $24 \mathrm{~h}$ after treatment ended) and also rescued the fragmented mitochondrial phenotype (Fig. 6A-C). The results obtained with mice and MEFs bearing SCA28-type mutations are consistent with perturbed substrate recognition by Afg312, leading to mitochondrial proteotoxicity through gradual accumulation of incorrectly processed or improperly degraded proteins. 
A

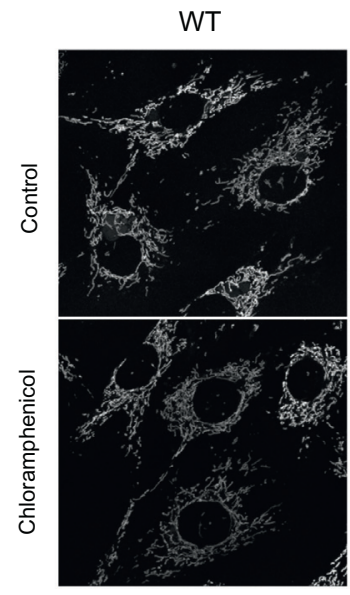

$\operatorname{Afg} 3 / 2^{M 665 R /+}$

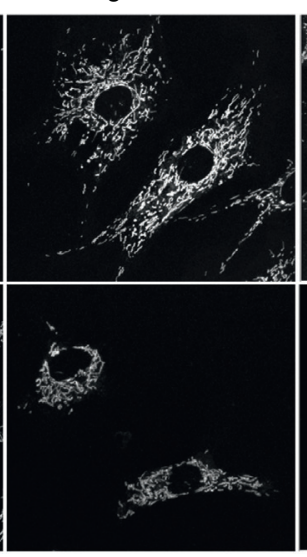

$\operatorname{Afg} 3 / 2^{M 665 R / M 665 R}$

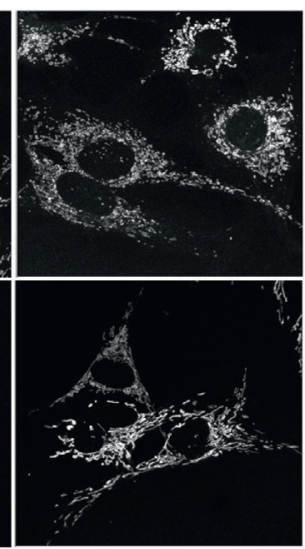

MEFs

B

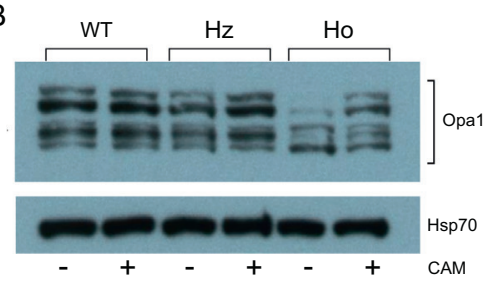

C

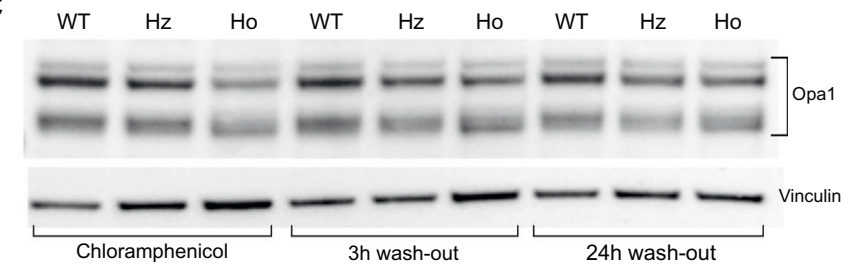

Fig. 6. Rescue of mitochondrial dynamics by chloramphenicol treatment.

(A). Representative images obtained by live confocal microscopy showing mitochondrial morphology in MEFs stained with mitoRED probe. Cells were untreated or treated with $200 \mu \mathrm{g} / \mathrm{ml}$ chloramphenicol. (B). Immunoblot analysis of total lysates obtained from MEFs untreated or treated with CAM; Hsp70 was used as loading control. (C). Immunoblot analysis of MEF total lysates treated with chloramphenicol, $3 \mathrm{~h}$ and $24 \mathrm{~h}$ after CAM wash-out. Vinculin was used as loading control.

\section{Discussion}

Since the discovery of AFG3L2 as the causative gene of SCA28, understanding the molecular basis of this neurodegenerative disease has been a major goal. The route we selected here was to produce a genetically engineered mouse that mirrored the human disease as closely as possible. At the outset, the idea of modelling a mild human adult-onset ataxia in mice was not without risk, as their short life-span might not allow the time needed for the phenotype to develop.

A number of murine models targeting Afg3l2 have been developed, with genetic modifications not present in human SCA28. These models vary mechanistically from haploinsufficiency $\left(A f g 3 l 2^{E m v 66 /+}\right)$ to complete deficiency in toto (Afg3l2 ${ }^{\text {Emv66/Emv66 }}, A f g 3 l 2^{\text {par/par }}$ ) to selective deficiency in Purkinje cells ( $A f g 3 l 2^{P C-\mathrm{KO}}$ ). These models have been successful in creating severe disease phenotypes that recapitulate in part the neurological manifestations found in SCA28 (Almajan et al., 2012; Maltecca et al., 2008; Maltecca et al., 2009; Martinelli et al., 2009). However, none of the current models harbour a single-copy patient mutation. Thus the new knock-in mouse model reported here, in which one allele of Afg3l2 is modified to carry the c.1994 T > G:p.Met666Arg point mutation (p.Met665Arg in mouse) derived from a SCA28 patient, can claim to genetically mirror the situation in humans.

How faithfully does the murine phenotype of the Afg $32^{M 665 R /+}$ mouse recapitulate the human disease? In patients, the ataxic phenotype generally starts in adulthood and progresses very slowly (patients are usually not confined to a wheelchair until into their sixties or seventies). That our knock-in mice did develop a mild form of ataxia at 18 months of age is consistent with the human pattern of disease, just as the late-onset is consistent with the differences in human-mouse chronobiology. The mouse motor phenotype was detected by the beam walking test. This is the most sensitive method to reveal fine coordination defects and motor ataxia due to cerebellar dysfunction. However, the other motor tests were non-discriminatory. One possible interpretation is that although early cerebellar damage may be present, it does not yet affect most motor functions.

How does the $A f g 3 l 2^{M 665 R /+}$ mouse compare to other mouse strains with modified Afg3l2? The knock-in mouse has a milder phenotype than that of the haploinsufficient strains: (i) in Afg3l2 ${ }^{\text {Emv66/+ }}$ mice, gait alterations develop at 4 months (Maltecca et al., 2009); (ii) in $A f g 3 l 2^{P C-K O}$ mice, gait unsteadiness develops at 6-8 weeks (Almajan et al., 2012), and (iii) in $S p g 7^{-1-} A f g 3 l 2^{E m v 66 /+}$ mice, altered coordination develops at 6 weeks (Martinelli et al., 2009). Thus, in terms of late-onset mild ataxia, the $A f g 3 l 2^{M 665 R /+}$ mouse appears to more faithfully represent the SCA28 phenotype.

In SCA28, there is MRI evidence of cerebellar atrophy with Purkinje cell loss in almost all patients analysed. Despite the detailed investigation, we found no evidence of cerebellar pathology in the knockin mice. On the other hand: 1) this is consistent with the mildness of the motor deficits observed; 2) severe ataxia may occur in the absence of signs of neurodegeneration (White et al., 2016); 3) although expression of Afg3l1 is low in cerebellum, we cannot exclude that even small amounts may attenuate the knock-in phenotype. This could be a future area of investigation. Purkinje cell numbers were normal, without the dark cell degeneration described in the Afg $322^{\text {Emv66/+ }}$ mice (Maltecca et al., 2009). We postulate that the most likely cause of motor incoordination in $A f g 3 l 2^{M 665 R /+}$ mice must be a qualitative rather that quantitative alteration of Purkinje cells. An indication of altered function was their tendency towards increased action potential firing. This tendency could be linked to impaired buffering of the ionic currents underlying the firing activity, and could represent an initial sign of Purkinje cell dysfunction, leading to their incapacity to depolarize correctly.

Purkinje cell dysfunction points to the crucial role of AFG3L2 in maintaining functional organelles and regulating mitochondrial dynamics (Koppen and Langer, 2007; Koppen et al., 2007). With their complex dendritic arborizations and synapses, Purkinje cells require a 
healthy and extensive mitochondrial network for ATP synthesis, axonal transport, and to buffer calcium influx. This network is maintained by a delicate balance between the opposing forces of mitochondrial fusion and fission. A particular feature of Purkinje cell mitochondria is that they have a longer lifespan than those of other tissues, making them more vulnerable to the cumulative effects of oxidative, proteotoxic, DNA and other forms of damage (Chen et al., 2007).

How does Afg312 - p.Met665Arg affect the function of the m-AAA protease and lead to mitochondrial dysfunction? We addressed this question using the mouse embryonic fibroblast model, which revealed various mitochondrial alterations. Firstly, there was evident increased mitochondrial fragmentation in homozygous Afg3l2 ${ }^{M 665 R / M 665 R}$ MEFs, whereas $A f g 3 l 2^{M 665 R /+}$ MEFs displayed an intermediate/tubular phenotype. Fragmentation of the mitochondrial network is known to be caused by an imbalance in proteolytic cleavage of Opa1, which together with the back-up protease Oma1, is one of the determinants of mitochondrial survival (Rugarli and Langer, 2012). Our findings of increased fragmentation were corroborated by biochemical evidence of overproduction of the short isoform of Opa1 (in particular S1 and S3 isoforms) in $A f g 3 l 2^{M 665 R / M 665 R}$ and $A f g 3 l 2^{M 665 R /+}$ MEFs. Extensive mitochondrial fragmentation was also detected in $A f g 32^{\text {Emv66/Emv66 }}$ MEFs (Maltecca et al., 2009). Proceeding from the MEF cellular model to the whole organism, we also observed impaired Opa1 processing in cerebellar lysates of 18-month old $A f g 3 l 2^{M 665 R /+}$ mice, suggesting this could be one of the early events in SCA28 pathogenesis, although patient mitochondria have yet to be analysed. Increased processing of Opa1 could also play an important role in the perinatal lethality of the homozygous knock-in mice. Opa1 is central for cardiac function, especially during cardiomyocyte differentiation and heart development (Wai et al., 2015). Like Opa1, Afg3l2 is also highly expressed in the developing heart (http://www.genecards.org/cgi-bin/carddisp.pl? gene $=$ AFG3L2\&keywords $=$ afg312), possibly linking the two proteases in the perinatal lethality of the knock-in mouse, which will be a future avenue of research.

Altered Opa1 cleavage has also been associated with enhanced apoptosis (Jiang et al., 2014; Rugarli and Langer, 2012), altered morphology of mitochondrial cristae, and decreased cell respiration and ATP production (Anand et al., 2014). This is consistent with the impaired bioenergetics observed in $A f g 3 l 2^{M 665 R / M 665 R}$ MEF mitochondria that revealed significantly reduced respiration and ATP synthesis. Altered Opa1 processing in Afg3l2 $2^{M 665 R / M 665 R}$ MEFs may be linked to the decrease in mitochondrial membrane potential compared to WT MEFs, which has been shown to induce activation of the ATP-independent zinc metalloprotease Oma1 which, in turn, promotes Opa1 proteolytic cleavage (Zhang et al., 2014). Indeed, increased pre-pro-Oma1 (60 kDa form) was detected in $A f g 32^{M 665 R / M 665 R}$ MEFs, supporting its activated state (Head et al., 2009; Korwitz et al., 2016).

Based on the results presented here, we hypothesize that Afg3l2 p.Met665Arg reduces the processing capability/rate of $m$-AAA, resulting in failure to turnover damaged or misfolded mitochondrial proteins, together with the accumulation of de novo synthesized proteins in organello. The burden of newly synthesized mitochondrial proteins may provoke inner membrane depolarization which, in association with protetoxic stress, activates the Oma1 protease. Consequently, Opa1 processing is impaired, fragmentation is enhanced and the mitochondrial network is disrupted, causing a functional alteration of the cell, and ultimately impairing neuronal functions (Fig. 7). Therefore, we decided to block mitochondrial de novo protein synthesis using the antibiotic chloramphenicol, a broad spectrum antibiotic that inhibits translation in both mitochondria and prokaryotes (Bulkley et al., 2010; Richter et al., 2015). By blocking mitochondrial protein synthesis, we were able to restore the correct balance between Opa1 isoforms and re-establish the mitochondrial network in Afg3l2 ${ }^{M 665 R / M 665 R}$ MEFs. This result demonstrates that the p.Met665Arg mutation in Afg312 affects the chaperone and proteolytic functions of the m-AAA complex, reducing protein quality surveillance in mitochondria. In agreement with this, bioinformatics analysis of AFG3L2 p.Met666Arg missense change showed that the electrostatic potential difference of the homohexamer complex central pore is altered with respect to wild-type AFG3L2, pointing to a reduction in the capacity of $m$-AAA to deliver substrates to the proteolytic chamber, or to a weaker interaction among monomers (Cagnoli et al., 2010). Chloramphenicol may also be affecting crosstalk between mitochondria and nucleus, as stalled translation might activate a retrograde signal to the nucleus, resulting in a compensatory pathway that inhibits Opa1 cleavage.

Experimental data involving the $m$-AAA protease must take into account that its roles are only partially understood. In mice, Afg311 (a pseudogene in humans), a third paralogue of Afg312 and Spg7, can modulate $m$-AAA function. Thus, at least five different types of $m$-AAA complexes are formed (Afg312 x 6; Afg311 x 6; Afg312 + Spg7; Afg312 + Afg311 and Afg311 + Spg7) (Koppen et al., 2007) and different hexamers may have distinct substrate or tissue specificities. For example, we know that Afg311 is the least expressed of the subunits in all tissues analysed, especially in cerebellum and brain (Sacco et al., 2010). In the presence of a mutated Afg312 with impaired protease activity, paraplegin and Afg311 subunits could be sequestered in nonfunctional hetero-hexameric complexes, reducing the amount of functional $m$-AAA complexes. In contrast, Afg3l2 deficiency would lead to the complete absence of protein, allowing Afg311 homo-hexamers and Afg311/Spg7 hetero-hexamers to form functional m-AAA complexes. Such a compensatory effect that has been demonstrated in $A f g 3 l 2^{P C-K O}$ mouse oligodendrocytes (Almajan et al., 2012).

In Afg3l2 $2^{M 65 R /+}$ mice, Afg3l2 synthesis is not impaired and a physiological number of m-AAA complexes should be formed, with wild-type and mutated subunits participating equally. We propose that a certain quantity of mutant Afg312 subunits can be tolerated, allowing normal $m$-AAA function to be maintained (up to a point). Thus we speculate that the heterozygous knock-in mice form a greater number of functional (or partially functional) $m$-AAA hexamers than in the heterozygous knockout mice, explaining the less severe phenotype. Indeed, all the previous mouse models strongly support a gene dosage effect of the three subunits (Afg312, Afg311 and Spg7) in determining the functional outcome of $m$-AAA hexamers (Ferreirinha et al., 2004; Maltecca et al., 2008; Maltecca et al., 2009; Martinelli et al., 2009; Wang et al., 2016). However, given that we ignore the m-AAA subunit stoichiometry and the reciprocal disposition of the subunits (alternate/continuous) in the complex, it remains a speculative hypothesis that warrants further experiment.

The role of Afg312 in Spg7 processing may also shed light on the effect of p.Met665Arg. Spg7 must be processed at two cleavage sites to activate its protease activity, the latter being dependent on Afg312/ Afg311 (Koppen et al., 2009). By electron microscopy, sciatic nerves of Afg $312^{M 665 R /+}$ mice show swollen mitochondria with disrupted cristae, corroborating the idea that the Afg312-paraplegin complex, highly represented in the peripheral nervous system, is also affected in our knock-in model (Koppen et al., 2007; Sacco et al., 2010).

In conclusion, we have investigated the molecular mechanisms leading to SCA28 with a newly developed knock-in mouse model which we believe better mimicks the human disease than previous models. We propose that mitochondrial proteostasis, leading to slow and toxic accumulation, is the pathogenic triggering event. Our findings open a novel therapeutic route, based on the modulation of mitochondrial translation, to be investigated in future studies.

Supplementary data to this article can be found online at https:// doi.org/10.1016/j.nbd.2018.10.018.

\section{Author contributions}

$\mathrm{CM}, \mathrm{EH}, \mathrm{AB}, \mathrm{FT}$ designed research studies, conducted experiments, acquired data, analysed data and wrote the manuscript. LI, UR, SG, FM, CC, RP, DIGM, VN, LM, GR, SG, FA (Arnaboldi), ED, EG, SC, EDG, EP, 


\section{+ m-AAA protease functional capacity}



Fig. 7. Pathogenic mechanisms triggering SCA28.

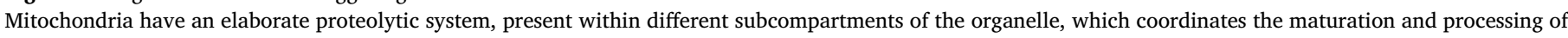

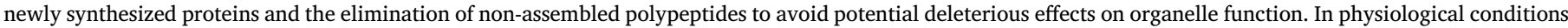

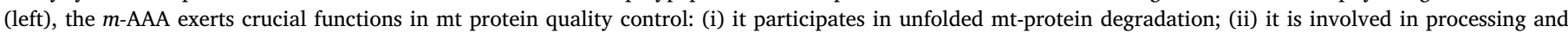

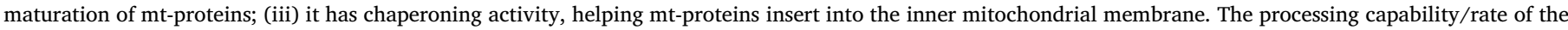

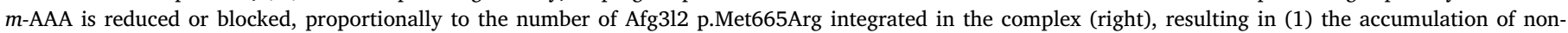

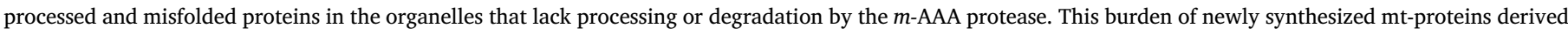

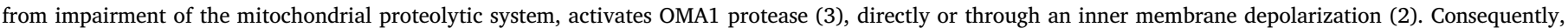

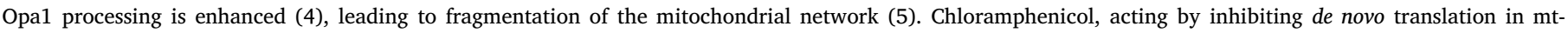

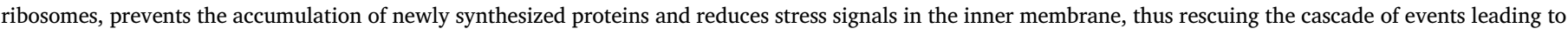
Opa1 enhanced processing and mt-fragmentation.

MF, ER, FA (Altruda), ET, GG, BJB, AMP conducted experiments and acquired data. A Brussino, EF wrote the manuscript. GC, FM made critical contributions to the discussion.

\section{Conflict of interest}

The authors have declared that no conflict of interest exists

\section{Acknowledgements}

This work was supported by the Telethon Foundation [grant number GGP12217 to AB and FT]; and by the Italian Ministry of Education, University and Research (EX60\% to AB; EX60\% to FT). CM and EG were supported by postdoc fellowships from the Umberto Veronesi Foundation.

\section{References}

Almajan, E.R., et al., 2012. AFG3L2 supports mitochondrial protein synthesis and Purkinje cell survival. J. Clin. Invest. 122, 4048-4058.

Almontashiri, N.A., et al., 2014. SPG7 variant escapes phosphorylation-regulated processing by AFG3L2, elevates mitochondrial ROS, and is associated with multiple clinical phenotypes. Cell Rep. 7, 834-847.

Anand, R., et al., 2014. The i-AAA protease YME1L and OMA1 cleave OPA1 to balance mitochondrial fusion and fission. J. Cell Biol. 204, 919-929.

Arlt, H., et al., 1996. The YTA10-12 complex, an AAA protease with chaperone-like ac tivity in the inner membrane of mitochondria. Cell 85, 875-885.

Arlt, H., et al., 1998. The formation of respiratory chain complexes in mitochondria is under the proteolytic control of the m-AAA protease. EMBO J. 17, 4837-4847.

Ashizawa, T., et al., 2018. Spinocerebellar ataxias: prospects and challenges for therapy development. Nat. Rev. Neurol. 14, 590-605.

Atorino, L., et al., 2003. Loss of m-AAA protease in mitochondria causes complex I deficiency and increased sensitivity to oxidative stress in hereditary spastic paraplegia. J. Cell Biol. 163, 777-787.
Baker, M.J., et al., 2014. Stress-induced OMA1 activation and autocatalytic turnover regulate OPA1-dependent mitochondrial dynamics. EMBO J. 33, 578-593.

Brussino, A., et al., 2018. Spinocerebellar Ataxia Type 28.

Bulkley, D., et al., 2010. Revisiting the structures of several antibiotics bound to the bacterial ribosome. Proc. Natl. Acad. Sci. U. S. A. 107, 17158-17163.

Cagnoli, C., et al., 2006. SCA28, a novel form of autosomal dominant cerebellar ataxia on chromosome 18p11.22-q11.2. Brain 129, 235-242.

Cagnoli, C., et al., 2010. Missense mutations in the AFG3L2 proteolytic domain account for approximately $1.5 \%$ of European autosomal dominant cerebellar ataxias. Hum. Mutat. 31, 1117-1124.

Carulli, D., et al., 2002. Regenerative and survival capabilities of Purkinje cells overexpressing c-Jun. Eur. J. Neurosci. 16, 105-118.

Casari, G., et al., 1998. Spastic paraplegia and OXPHOS impairment caused by mutations in paraplegin, a nuclear-encoded mitochondrial metalloprotease. Cell 93, 973-983.

Charif, M., et al., 2015. A novel mutation of AFG3L2 might cause dominant optic atrophy in patients with mild intellectual disability. Front. Genet. 6, 311.

Chen, H., et al., 2007. Mitochondrial fusion protects against neurodegeneration in the cerebellum. Cell 130, 548-562.

Colavito, D., et al., 2017. Non-syndromic isolated dominant optic atrophy caused by the p.R468C mutation in the AFG3 like matrix AAA peptidase subunit 2 gene. Biomed. Rep. 7, 451-454.

Consolato, F., et al., 2018. m-AAA and i-AAA complexes coordinate to regulate OMA1, the stress-activated supervisor of mitochondrial dynamics. J. Cell Sci. 131.

Copeland, N.G., et al., 2001. Recombineering: a powerful new tool for mouse functional genomics. Nat. Rev. Genet. 2, 769-779.

Dalbey, R.E., et al., 2012. Membrane proteases in the bacterial protein secretion and quality control pathway. Microbiol. Mol. Biol. Rev. 76, 311-330.

Di Bella, D., et al., 2010. Mutations in the mitochondrial protease gene AFG3L2 cause dominant hereditary ataxia SCA28. Nat. Genet. 42, 313-321.

Ding, B., et al., 2018. Dissecting Substrate Specificities of the Mitochondrial AFG3L2 Protease. Biochemistry 57, 4225-4235.

Edener, U., et al., 2010. Early onset and slow progression of SCA28, a rare dominant ataxia in a large four-generation family with a novel AFG3L2 mutation. Eur. J. Hum. Genet. 18, 965-968.

Ehses, S., et al., 2009. Regulation of OPA1 processing and mitochondrial fusion by m-AAA protease isoenzymes and OMA1. J. Cell Biol. 187, 1023-1036.

Ferreirinha, F., et al., 2004. Axonal degeneration in paraplegin-deficient mice is associated with abnormal mitochondria and impairment of axonal transport. J. Clin. Invest. 113, 231-242.

Gerdes, F., et al., 2012. Mitochondrial AAA proteases - Towards a molecular 
understanding of membrane-bound proteolytic machines. Biochim. Biophys. Acta 1823, 49-55.

Geuna, S., 2000. Appreciating the difference between design-based and model-based sampling strategies in quantitative morphology of the nervous system. J. Comp. Neurol. 427, 333-339.

Geuna, S., et al., 2000. Verification of the two-dimensional disector, a method for the unbiased estimation of density and number of myelinated nerve fibers in peripheral nerves. Ann. Anat. 182, 23-34.

Ghelli, A., et al., 2013. The cytochrome b p.278Y > C mutation causative of a multisystem disorder enhances superoxide production and alters supramolecular interactions of respiratory chain complexes. Hum. Mol. Genet. 22, 2141-2151.

Goldowitz, D., Moran, T.H., Wetts, R., 1992. Mouse Chimeras in the Study of Genetic and Structural Determinants of Behavior: Techniques for the Genetic Analysis of Brain and Behavior. Elsevier, Amsterdam.

Head, B., et al., 2009. Inducible proteolytic inactivation of OPA1 mediated by the OMA1 protease in mammalian cells. J. Cell Biol. 187, 959-966.

Hoxha, E., et al., 2013. Motor dysfunction and cerebellar Purkinje cell firing impairment in Ebf2 null mice. Mol. Cell. Neurosci. 52, 51-61.

Iommarini, L., et al., 2014. Different mtDNA mutations modify tumor progression in dependence of the degree of respiratory complex I impairment. Hum. Mol. Genet. 23, 1453-1466.

Jiang, X., et al., 2014. Activation of mitochondrial protease OMA1 by Bax and Bak promotes cytochrome c release during apoptosis. Proc. Natl. Acad. Sci. U. S. A. 111, $14782-14787$.

Koppen, M., Langer, T., 2007. Protein degradation within mitochondria: versatile activities of AAA proteases and other peptidases. Crit. Rev. Biochem. Mol. Biol. 42, 221-242.

Koppen, M., et al., 2007. Variable and tissue-specific subunit composition of mitochondrial m-AAA protease complexes linked to hereditary spastic paraplegia. Mol. Cell. Biol. 27, 758-767.

Koppen, M., et al., 2009. Autocatalytic processing of m-AAA protease subunits in mitochondria. Mol. Biol. Cell 20, 4216-4224.

Korwitz, A., et al., 2016. Loss of OMA1 delays neurodegeneration by preventing stressinduced OPA1 processing in mitochondria. J. Cell Biol. 212, 157-166.

Kremmidiotis, G., et al., 2001. Molecular and functional analyses of the human and mouse genes encoding AFG3L1, a mitochondrial metalloprotease homologous to the human spastic paraplegia protein. Genomics 76, 58-65.

Larsen, J.O., 1998. Stereology of nerve cross sections. J. Neurosci. Methods 85, 107-118.

Lek, M., et al., 2016. Analysis of protein-coding genetic variation in 60,706 humans. Nature 536, 285-291.

Liesa, M., et al., 2009. Mitochondrial dynamics in mammalian health and disease. Physiol. Rev. 89, 799-845.

Lobbe, A.M., et al., 2014. A novel missense mutation in AFG3L2 associated with late onset and slow progression of spinocerebellar ataxia type 28. J. Mol. Neurosci. 52, 493-496.

MacVicar, T., Langer, T., 2016. OPA1 processing in cell death and disease - the long and short of it. J. Cell Sci. 129, 2297-2306.

Maltecca, F., et al., 2008. The mitochondrial protease AFG3L2 is essential for axonal development. J. Neurosci. 28, 2827-2836.

Maltecca, F., et al., 2009. Haploinsufficiency of AFG3L2, the gene responsible for spinocerebellar ataxia type 28 , causes mitochondria-mediated Purkinje cell dark degeneration. J. Neurosci. 29, 9244-9254.

Maltecca, F., et al., 2012. Respiratory dysfunction by AFG3L2 deficiency causes decreased mitochondrial calcium uptake via organellar network fragmentation. Hum. Mol. Genet. 21, 3858-3870.

Mancini, C., et al., 2011. Gene-targeted embryonic stem cells: real-time PCR assay for estimation of the number of neomycin selection cassettes. Biol. Proc. Online. 13, 10.

Mariotti, C., et al., 2008. Spinocerebellar ataxia type 28: a novel autosomal dominant cerebellar ataxia characterized by slow progression and ophthalmoparesis. Cerebellum 7, 184-188.

Martinelli, P., et al., 2009. Genetic interaction between the m-AAA protease isoenzymes reveals novel roles in cerebellar degeneration. Hum. Mol. Genet. 18, 2001-2013.

Musova, Z., et al., 2014. A novel frameshift mutation in the AFG3L2 gene in a patient with spinocerebellar ataxia. Cerebellum 13, 331-337.

Nibbeling, E.A.R., et al., 2017. Exome sequencing and network analysis identifies shared mechanisms underlying spinocerebellar ataxia. Brain 140, 2860-2878.

Nolden, M., et al., 2005. The m-AAA protease defective in hereditary spastic paraplegia controls ribosome assembly in mitochondria. Cell 123, 277-289.

Patron, M., et al., 2018. m-AAA proteases, mitochondrial calcium homeostasis and neurodegeneration. Cell Res. 28, 296-306.

Quiros, P.M., et al., 2012. Loss of mitochondrial protease OMA1 alters processing of the GTPase OPA1 and causes obesity and defective thermogenesis in mice. EMBO J. 31, 2117-2133.

Richter, U., et al., 2015. Quality control of mitochondrial protein synthesis is required for membrane integrity and cell fitness. J. Cell Biol. 211, 373-389.

Rugarli, E.I., Langer, T., 2012. Mitochondrial quality control: a matter of life and death for neurons. EMBO J. 31, 1336-1349.

Sacco, T., et al., 2010. Mouse brain expression patterns of Spg7, Afg311, and Afg312 transcripts, encoding for the mitochondrial m-AAA protease. BMC Neurosci. 11, 55.

Schuermann, M., 1990. An expression vector system for stable expression of oncogenes. Nucleic Acids Res. 18, 4945-4946.

Sharpless, N., 2006. Preparation and Immortalization of Primary Murine Cells. Cell Biol. 1, 223-228.

Smeets, C.J., Verbeek, D.S., 2014. Cerebellar ataxia and functional genomics: Identifying the routes to cerebellar neurodegeneration. Biochim. Biophys. Acta 1842, 2030-2038.

Smets, K., et al., 2014. Partial deletion of AFG3L2 causing spinocerebellar ataxia type 28. Neurology 82, 2092-2100.

Sorrentino, V., et al., 2017. Enhancing mitochondrial proteostasis reduces amyloid-beta proteotoxicity. Nature 552, 187-193.

Szpisjak, L., et al., 2017. Neurocognitive Characterization of an SCA28 Family Caused by a Novel AFG3L2 Gene Mutation. Cerebellum 16, 979-985.

Thaung, C., et al., 2002. Presence of visual head tracking differentiates normal sighted from retinal degenerate mice. Neurosci. Lett. 325, 21-24.

Wai, T., et al., 2015. Imbalanced OPA1 processing and mitochondrial fragmentation cause heart failure in mice. Science 350 , aad0116.

Wang, S., et al., 2016. The mitochondrial m-AAA protease prevents demyelination and hair greying. PLoS Genet. 12, e1006463.

White, J.J., et al., 2016. Pathogenesis of severe ataxia and tremor without the typical signs of neurodegeneration. Neurobiol. Dis. 86, 86-98.

Zhang, K., et al., 2014. Membrane depolarization activates the mitochondrial protease OMA1 by stimulating self-cleavage. EMBO Rep. 15, 576-585.

Zuhlke, C., et al., 2015. Spinocerebellar ataxia 28: a novel AFG3L2 mutation in a German family with young onset, slow progression and saccadic slowing. Cerebellum Ataxias. $2,19$. 